\title{
FUZZY REVERSE LOGISTICS INVENTORY MODEL OF SMART ITEMS WITH TWO WAREHOUSES OF A RETAILER CONSIDERING CARBON EMISSIONS
}

\author{
Subhash Kumar ${ }^{1}$, Biswajit Sarkar ${ }^{2, *}$ And Ashok Kumar ${ }^{1}$
}

\begin{abstract}
Running the business smoothly for protecting the environment is a significant challenge, on which industries are trying something to do at their level best. Reverse logistics play an important role in system design by reducing environmental consequences and increasing economic and social impacts. Given the recent fluctuations of the market, the production cost and ordering cost are considered triangular fuzzy numbers in this study. Customers' demand is met at the right time, and there is no shortage of items; thus, attention can be paid to two warehouses of a retailer. The setup costs Purchasing costs and deterioration costs of this system are affected by the learning effects, which lead to a decrease in the total cost. Inflation is a significant problem in the market because manufacturing, remanufacturing, and retailers are all affected. This study proposes a reverse logistics system model so that customers can resolve their complaints about defective items and carbon emissions under two warehouses. Numerical results show that the fuzzy model is more economically beneficial than the crisp model, finds that the crisp and fuzzy model saw a difference of $0.34 \%$ in total cost. Two numerical examples illustrate this study, and a sensitivity analysis is performed using tables and graph.
\end{abstract}

Mathematics Subject Classification. 90B05, 90B30, 90C30, 90C70.

Received November 27, 2020 Accepted April 5, 2021.

\section{INTRODUCTION}

Comprehensive strategies to manage and promote green activities through the reverse supply chain have been developed and adopted by significant producers worldwide. Thus, the quality of the environment is not affected, and carbon emissions are controlled. There has been an increased interest in reverse logistics (RL) over the last few years. It has seen that all customers tend to buy products after looking at their warranty, guaranty, returnable period.

Apart from this, to make a business successful, the retailer should also have a system to keep enough items, not to face any problem when many goods are ordered for delivery. Customers' demands can deliver at the right time, maintaining a good impression in the market. In this regard, the role of more than one warehouse is significant. The use of rented warehouses by the retailer has made the cost somewhat expensive, but it maintains the market continuity in the supply chain process. Sarkar et al. [30] discussed a remanufacturing

Keywords. Remanufacturing, two warehouses, carbon emission, learning effect, fuzzy environment.

1 Department of Mathematics, Meerut College Meerut, Uttar Pradesh, India.

2 Department of Industrial Engineering, Yonsei University, 50 Yonsei-ro, Sinchon-dong, Seodaemun-gu, Seoul 03722,

South Korea.

*Corresponding author: bsbiswajitsarkar@gmail.com 
model in environmental impacts of transportation in this direction. Simultaneously, Saxena et al. [37] analyzed a remanufacturing inventory model base for the primary and secondary markets. Jawla and Singh [12] examined in reverse logistics model with the influence of inflation. After that, Ullah et al. [43] examined an optimal remanufacturing system and reusable packaging capacity for single and multi-retailer closed-loop supply chain models under stochastic demand and return rate. Singh et al. [36] introduced a remanufacturing model that further introduces learning on costs. These costs decrease due to the previous cycle's learning influence. Rani et al. [25] found that consumers are becoming more mindful about protecting the environment and favor goods produced with low carbon. Customers observe to pay much more for goods have with low-carbon emission technology in some cases. Moreover, Sarkar et al. [32] model developed a model that reduces waste and controls pollution to save the environment easily.

To the best of the authors' knowledge, no reverse fuzzy logistics model has been developed considering two warehouses. This study complements the research gap in two warehouses of a retailer considering carbon emissions, inflation, and learning effect under a fuzzy reverse logistics model. Reverse logistics (RL) attempts to maintain customer satisfaction, and another issue is the gathering of used products from customers, which is related to the environment and supply chain management. Thus, RL helps build a green supply chain management with reusing products, which is also a part of waste management. According to the actual market situation, an order cost and production cost are considered the fuzzy triangular number. The impact of inflation and time value is also considered to make it more practical and suitable for the present environment. This study aims to combine carbon emission and waste reduction items with two warehouses of a retailer within the fuzzy reverse logistics management and minimize the total cost in a crisp and fuzzy environment with a learning effect. The current paper is organized as follows: In Section 2, the literature review is presented in this section. Section 3, notations and assumptions of that model are provided. Section 4 deals with the mathematical model and procedure for the optimal solution. Section 5 is devoted to numerical analysis. Section 6 explains some parameters' sensitivity, and Section 7 describes the conclusions and future research directions.

\section{Literature REVIEW}

Recently, some research has been conducted on this topic. According to Chouinard [5], RL operations are related to collecting and recycling consumer products and the reuse of surplus items. New approaches and information support services have been recommended for better monitoring and management of these operations. Bernon and Cullen [3] established a structure for handling RL by following the three management methods of integration, collaboration, and evaluation. Liao [18] developed a nonlinear programming model for an RL network architecture. The RL concept for multi-echelon was considered. It maximized the overall value of items returned for repair, remanufacturing, recycling, reuse, or incineration/landfill. Singh and Sharma [35] analyzed an RL model considering logistics in an inflationary situation. They considered limited and variable production and remanufacturing rates. Sarkar et al. [31] established a closed-loop supply chain (CLSM) model with a single supplier, a single producer, and multiple retailers under budget and storage constraints. They reduced certain losses by reworking an incentive modeling strategy at each level of a cycle period by identifying opportunities. Sarkar [29] established a concept for making such a form of a multi-stage production system. During each cycle, defective goods could be reworked while eliminating shortages; on the other hand, shortages could be reworked in each process after the last cycle. Green service was investigated by Maric [22] as a technique to promote agile and efficient supply chain management (SCM). They discussed drivers and obstacles faced by computer and electronics business experts when deploying RL services. Simultaneously, they attempted to reveal how electronics companies' practitioners can accomplish supportable end-of-life item recovery using green technology and RL services. Rani et al. [26] established an RL model with demand is directly dependent on the credit period. Rani et al. [27] examined the electronics sector, which uses RL to acquire and refurbish used products. Such goods are sold at a discounted price on the market. Safdar [33] proposed a multi-goal RL strategy concept. The proposed concept aimed to create a trade-off between opposing interests. Saxena et al. [38] established to examine two replenishment period methods concerning waste management. A model with 
incompatible primary and secondary business principles and sequential production/remanufacturing policies was proposed.

Under the carbon cap-and-trade (CCT) policy, Dye and Yang [7] evaluated a standard model used to market and default risk rates. Bai et al. [1] considered one producer and two rival retailers to study the effect of carbon emission reduction on a supply chain for declining goods under a vendor-managed inventory. They showed that the CCT policy and investment in green technology could reduce the carbon emissions produced by manufacturing and inventory keeping in this scheme. Huang et al. [11] investigated the impact of climate policies and green technology on the integrated inventory of a two-stage supply chain, taking into account carbon emissions during the processing, transport, and storage processes of goods. Mishra et al. [23] analyzed models with a sustainable EPQ carbon tax, no shortage cap, a partial backorder cap, and a full backorder cap with and without investment in green technologies.

Fuzziness expresses the actual market situation well. Wee et al. [44] introduced new inverse weight fuzzy nonlinear programming to formulate a fuzzy model. Thus, the benefits and returns on product investment under fuzzy demand and cost constraints were maximized. Over a known-finite period, Roy et al. [28] studied a singleproduct production-remanufacturing method. Two versions were independently formulated with constant and fuzzy damaged production. When a faulty rate was imprecise, the reputation measure of a fuzzy case considering fuzzy expectation could be obtained by the positive and negative inverse of the fuzzy objective function. Kumar et al. [16] derived an approach in which randomness was calculated using a probabilistic assumption to scalarize the fuzzy stochastic joint overall related cost (JTRC). Khan and Dev [13] proposed a model in a mixed fuzzy random framework.

Considering the annual consumer demand to be a constant fuzzy random variable following a normal distribution, an assortment of these two uncertainties (fuzziness and randomness) was achieved. Noh and Kim [24] analyzed a situation under greenhouse gas pollution legislation between a single producer and several retailers with minimal capital for many goods categories. In their study, they considered a company that manufactures goods and ships them after inspections. Demand may either be defined or have some ambiguity for specific items and can be better expressed using a fuzzy number. Tayyab et al. [39] implemented an incomplete multi-stage production model, taking into account the flawed proportion of the manufacturing process and the unpredictable market for commodities. To control the complexity of the demand results, the fuzzy theory was used, and the center of gravity method was used to defuzzify the function. Tayyab et al. [40] presented an economic output quantity model with unclear demand and process details in a multi-stage manufacturing process. The decomposition rule and the fuzzy theory signed distance approach were applied to manage this production process's instability. Singh and Singh [34] discussed a joint framework with a seasonal trend demand rate of ramp form for expiring goods with the retailer's random lead time in a fuzzy system, considering the impact of inflation and money's time value.

Because of insufficient space, vast numbers of products should not be kept in an owned warehouse. To preserve the extra or surplus quantity of items, other storage facilities are therefore necessary. The classical inventory models studied by Chung et al. [6] typically assume that the usable warehouse has an infinite capacity. There are also variables in many realistic cases, such as immediate price discounts that allow manufacturers to acquire an inventory capability that exceeds their warehouse limit. In this scenario, retailers can rent other warehouses for business needs. Liao et al. [19] stated that rented warehouse (RW) stocks could be shipped to an owned warehouse (OW) via a consistent pattern of release, but transport costs were overlooked. In an RW, the holding expenditure exceeds that of an OW. It is assumed that the deterioration rate of an RW is equivalent to that of an OW. Manna et al. [20] analyzed a retailer's procurement of a manufacturer's large number and low-quality goods to market products to consumers from their finite capacity showrooms. The retailer employed a secondary warehouse with an unlimited capacity to store the excess quantity of excellent quality goods on a rental basis.

Majumder et al. [21] evaluated two warehouse inventory (TWI) strategies with a wholesaler for a credit period on a finite preparation horizon. Tiwari et al. [41] investigated a TWI model for declining goods with partial backlogging and a stock-dependent demand. Yu [45] established an inventory model consisting of one retailer and one seller with a two-warehouse ecosystem to manage a two-echelon system with deteriorating loss and 
TABLE 1. Author's contribution ( $\sqrt{ }$-include, $\boldsymbol{x}$ - exclude).

\begin{tabular}{lllllll}
\hline \hline Author (s) & Model & $\begin{array}{l}\text { Two } \\
\text { warehouses }\end{array}$ & $\begin{array}{l}\text { Learning } \\
\text { effect }\end{array}$ & $\begin{array}{l}\text { Carbon } \\
\text { emissions }\end{array}$ & Inflation & $\begin{array}{l}\text { Fuzzy } \\
\text { environment }\end{array}$ \\
\hline Jawla and Singh [12] & SCM under RP & $\boldsymbol{x}$ & $\sqrt{ }$ & $\boldsymbol{x}$ & $\sqrt{ }$ & $\boldsymbol{x}$ \\
Sarkar et al [30] & SCM under RP & $\boldsymbol{x}$ & $\boldsymbol{x}$ & $\sqrt{ }$ & $\boldsymbol{x}$ & $\boldsymbol{x}$ \\
Singh et al. [36] & SCM under RP & $\boldsymbol{x}$ & $\sqrt{ }$ & $\boldsymbol{x}$ & $\boldsymbol{x}$ & $\boldsymbol{x}$ \\
Sarkar et al. [32] & SCM & $\boldsymbol{x}$ & $\boldsymbol{x}$ & $\sqrt{ }$ & $\boldsymbol{x}$ & $\boldsymbol{x}$ \\
Rani et al. $[25]$ & SCM under RP & $\boldsymbol{x}$ & $\boldsymbol{x}$ & $\sqrt{ }$ & $\boldsymbol{x}$ & $\sqrt{ }$ \\
Ullah et al. $[43]$ & SCM under RP & $\boldsymbol{x}$ & $\boldsymbol{x}$ & $\boldsymbol{x}$ & $\boldsymbol{x}$ & $\boldsymbol{x}$ \\
This study & SCM under RP & $\sqrt{ }$ & $\sqrt{ }$ & $\sqrt{ }$ & $\sqrt{ }$ & $\sqrt{ }$ \\
\hline
\end{tabular}

Notes. SCM means supply chain management, RP means remanufacturing process.

shortage back-ordering. The stock price of the rented warehouse decreased over time. Under the assumption of shortages and partial backlogging underpayment delay, Chakrabarty et al. [4] examined TWI models for a single declining product. Also, they integrated the impact of inflation and time demand on revenue. Marketers typically want to find a store in a widespread marketing location. Thus, owing to inadequate space in a typical marketplace, extra storerooms are required. The advance payment plan made up to $\mathrm{n}$ times before acquiring the goods by equal installments was also considered. Khan et al. [14] developed a TWI model for aging goods with an automated payment system using all these collaboration ideas. Ghiamia and Beullens [8] examined stock-dependent demand in the case of declining goods for a retailer with TWI, where the retailer attempts to achieve the optimum integrated procurement strategy by cooperation with a supplier. The advantages of both the continuous resupply approach and the widely used approach have been established in the OW and RW literature. Khan et al. [15] found that the deterioration of OWs began sooner than that of RWs, as the latter still allowed for improved inventory storage and services.

Tsao and Sheen [42] reported that the revenue learning curve principle is considered in the cost of marketing. They aimed to overcome retailers' marketing and replenishment decisions with the revenue learning curve under retailer competition and promotional activity. Zhang et al. [46] focused on the success of a supply chain in a single maker-retailer setup. The unit manufacturing expense was found to be mutually influenced by the impact of the learning cost. A supply chain of stochastic product returns was investigated by Giri and Glock [9], considering worker expertise in understanding and forgetting created and checked returned products of a manufacturer. A CLSC model was developed by Giri and Masanta [10], with two manufacturers, one manufacturer, and one retailer. The consumer demand for the final product showed a linear dependency on the selling price and quality, and the manufacturing procedure was based on the theme of learning. Kumar et al. [17] developed a learning effect model for a green environment. Considering a two-level dual-channel supply chain, Batarfi et al. [2] examined the impact of learning and forgetting on manufacturing processes. Some author's contribution is showed in Table 1.

This study has adopted strategies to reduce waste and carbon emissions to make the environment less polluted. The manufacturer utilizes collected defective products inventory to remanufacturing process. This study developed here is a fuzzy RL inventory model with two warehouses of a retailer. The developed model was designed considering carbon emissions. A general flow of this model is depicted in Figure 1.

\section{Notation USED AND ASSUmptions MADE IN THE MODEL}

\section{The following notation is used in the model}

$\epsilon$ Carbon emissions.

$\omega$ Carbon emissions under the demand parameter.

$\tau$ Inflation rate. 


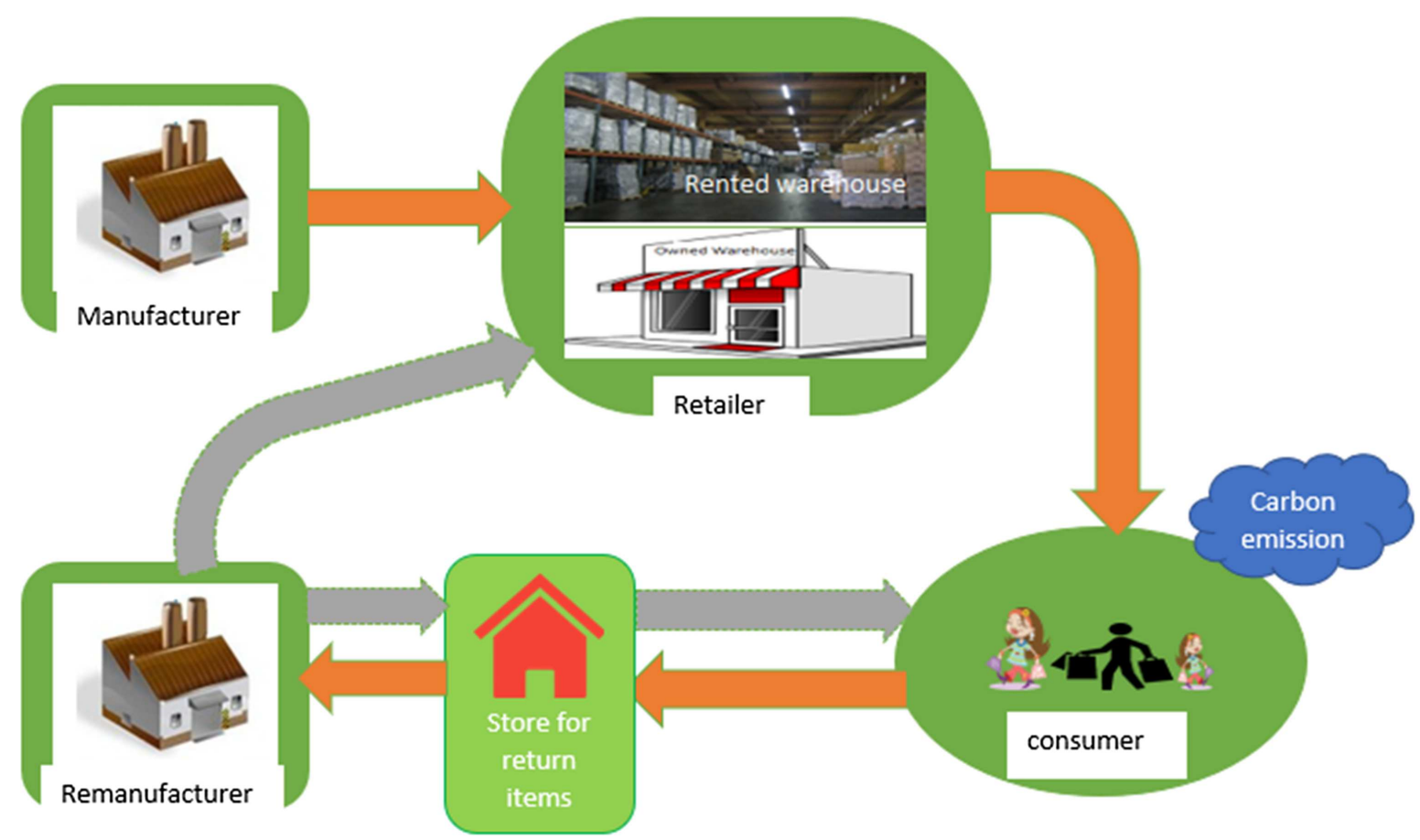

Figure 1. Process of the supply chain model.

\section{Manufacturing parameters}

$P_{M}$ Production rate.

$\mu_{1}$ Deterioration rate.

$\alpha_{1}, \alpha_{2}$ Demand rate.

$t_{M}$ Maximum inventory level time.

$t_{2 M}$ Time when the inventory level is zero.

$N$ Total number of manufacturing cycles in $T$.

$V_{M}$ Inventory level when $0 \leq t \leq t_{M}$.

$V_{2 M}$ Inventory level when $t_{M} \leq t \leq t_{2 M}$.

$S_{\mathrm{MC}}$ or $\left(S_{\mathrm{LMC}}+S_{1} / \Delta^{\mathrm{K}}\right)$ Effective learning setup cost.

$P_{\mathrm{MC}}$ Production cost.

$H_{\mathrm{MC}}$ Holding cost.

$D_{\mathrm{MC}}$ or $\left(D_{\mathrm{LMC}}+\frac{D_{1}}{\Delta^{\mathrm{K}}}\right)$ Effective learning deterioration cost.

\section{Retailer's parameters}

$C_{D}$ Demand rate.

$\mu_{2}$ Deterioration rate.

$M$ Highest storage capacity of OW.

$t_{r}$ Inventory level time when RW reaches zero.

$t_{2 r}$ Inventory level time of OW reaches zero.

$V_{\text {rr }}$ RW inventory level when $0 \leq t \leq t_{r}$.

$V_{2 \text { ro }}$ OW inventory level when $0 \leq t \leq t_{r}$.

$V_{3 \text { ro }}$ OW inventory level when $t_{r} \leq t \leq t_{2 r}$.

$O_{\mathrm{rC}}$ Ordering cost. 
$P_{\mathrm{rC}}$ or $\left(P_{\mathrm{LrC}}+\frac{P_{1}}{\Delta^{\mathrm{K}}}\right)$ Effective learning purchasing cost.

$H_{\mathrm{rrC}}$ Holding cost of RW.

$H_{\text {roC }}$ Holding cost of OW.

$D_{\mathrm{rC}}$ or $\left(D_{\mathrm{LrC}}+\frac{D_{2}}{\Delta^{\mathrm{K}}}\right)$ Effective learning deterioration cost.

\section{Remanufacturing parameters}

$P_{R}$ Reproduction rate.

$\mu_{1}$ Deterioration rate.

$\alpha_{1}, \alpha_{2}$ Demand rate.

$t_{R}$ Maximum inventory level time.

$T$ Total cycle time.

$V_{R}$ Inventory level during the period $0 \leq t \leq t_{R}$.

$V_{2 R}$ Inventory level during the period $t_{R} \leq t \leq T$.

$S_{\mathrm{RC}}$ or $\left(S_{\mathrm{LRC}}+\frac{S_{2}}{\Delta^{\mathrm{K}}}\right)$ Effective learning setup cost.

$P_{\mathrm{RC}}$ Production cost.

$H_{\mathrm{RC}}$ Holding cost.

$D_{\mathrm{RC}}$ or $\left(D_{\mathrm{LRC}}+\frac{D_{3}}{\Delta^{\mathrm{K}}}\right)$ Effective learning deterioration cost.

$C_{\mathrm{RC}}$ Collection cost.

$\rho$ Returned rate.

\section{Assumptions}

(1) Products are returned at the rate $\rho$, which are then collected and transported back to the manufacturer. The learning effect is applied to all the systems' deterioration costs, and the manufacturing and remanufacturing costs are set up.

(2) Order cost and production cost are considered as triangular fuzzy numbers.

(3) The time point of OW is double the time point of RW.

(4) Lead time is negligible.

(5) Returned products are recovered at the rate $\sigma$, while the waste products are recycled.

(6) OW has a finite capacity, whereas RW has an infinite capacity.

(7) The retailer stores the goods first in the owned warehouse and then in the rented warehouse and consumes the goods first from the rented warehouse and then from the owned warehouse.

\section{Mathematical MODEL}

In this model, it is assumed that manufacturing and remanufacturing occur simultaneously. However, they have different cycle times. One cycle consists of a single remanufacturing cycle and multiple manufacturing cycles and is represented as $T$. The remanufactured products have the same market and have the same quality as those of the new products.

\section{Manufacturing cycle}

The production begins at time $t=0$ and continues till $t_{M}$. The production rate is given by $P_{M}$. The inventory level decreases owing to the demand $\alpha_{1}+\alpha_{2} t$ and deterioration $\mu_{1}$. At $t=t_{M}$ sufficient inventory is accumulated, and production is stopped. The accumulated inventory continues to meet the demand $t_{2 M}$. At this time, the inventory becomes 0 , and the production cycle begins again (see Fig. 2).

$$
\frac{\mathrm{d} V_{M}}{\mathrm{~d} t}=P_{M}-\left(\alpha_{1}+\alpha_{2} t\right)-\mu_{1} V_{M}, \quad 0 \leq t \leq t_{M}
$$

with the boundary condition $V_{M}(0)=0$.

$$
\frac{\mathrm{d} V_{2 M}}{\mathrm{~d} t}=-\left(\alpha_{1}+\alpha_{2} t\right)-\mu_{1} V_{2 M}, \quad t_{M} \leq t \leq t_{2 M}
$$




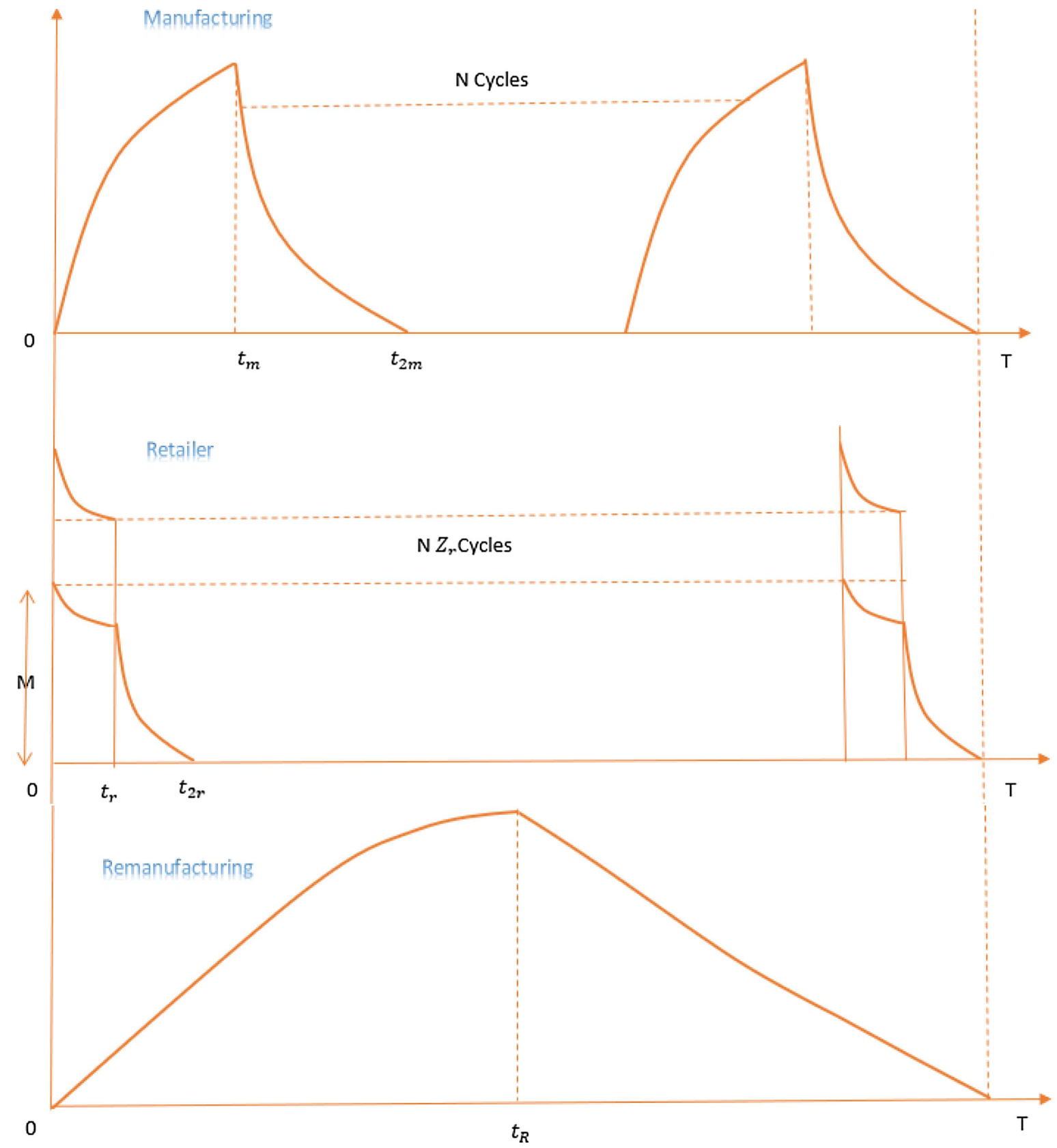

FiguRE 2. Inventory level of the supply chain model. 
with the boundary condition $V_{2 M}\left(t_{2 M}\right)=0$.

Then, the solutions of both equations are

$$
\begin{aligned}
V_{M} & =\frac{e^{-\mu_{1} t}}{\mu_{1}^{2}}\left[\left\{\left(P_{M}-\left(\alpha_{1}+\alpha_{2} t\right)\right) \mu_{1}+\alpha_{2}\right\} e^{\mu_{1} t}-\left\{\left(P_{M}-\alpha_{1}\right) \mu_{1}+\alpha_{2}\right\}\right] \\
V_{2 M} & =\frac{e^{-\mu_{1} t}}{\mu_{1}^{2}}\left[\left(\alpha_{2}-\left(\alpha_{1}+\alpha_{2} t\right) \mu_{1}\right) e^{\mu_{1} t}+\left(\left(\alpha_{1}+\alpha_{2} t_{2 M}\right) \mu_{1}-\alpha_{2}\right) e^{\mu_{1} t_{2 M}}\right] .
\end{aligned}
$$

Assuming that there exist $N$ manufacturing cycles in one overall cycle, $t_{2 M}$ is given by $t_{2 M}=\frac{T}{N}$.

\section{Retailer cycle}

Each manufacturing and remanufacturing cycle consists of multiple retailer cycles. In RW, the stock decline over the interval $0 \leq t \leq t_{r}$ and is related to the mutual impact of both the demand and deterioration $\mu_{2}$ and the decline in the inventory level in OW due to deterioration $\mu_{2}$ alone. In OW, the inventory level over the interval $t_{r} \leq t \leq t_{2 r}$ is related to the mutual impact of both demand and deterioration. Let this study assume that the customer demand $\left(C_{D}-\omega \epsilon\right)$ is a carbon-dependent demand.

$$
\begin{aligned}
\frac{\mathrm{d} V_{\mathrm{rr}}}{\mathrm{d} t} & =-\left(C_{D}-\omega \epsilon\right)-\mu_{2} V_{\mathrm{rr}}, & & 0 \leq t \leq t_{r} \\
V_{\mathrm{rr}}(0) & =0 & & \\
\frac{\mathrm{d} V_{2 \mathrm{ro}}}{\mathrm{d} t} & =-\mu_{2} V_{2 \mathrm{ro}}, & & 0 \leq t \leq t_{r} \\
V_{2 \mathrm{ro}}(0) & =M & & \\
\frac{\mathrm{d} V_{3 \mathrm{ro}}}{\mathrm{d} t} & =-\left(C_{D}-\omega \epsilon\right)-\mu_{2} V_{3 \mathrm{ro}}, & & t_{r} \leq t \leq t_{2 r} \\
V_{3 \mathrm{ro}}\left(t_{2 r}\right) & =0 . & &
\end{aligned}
$$

Then, the solutions for these equations are

$$
\begin{aligned}
V_{\mathrm{rr}} & =\frac{\left(C_{D}-\omega \epsilon\right)}{\mu_{2}} e^{-\mu_{2} t}\left[-e^{\mu_{2} t}+1\right] \\
V_{2 \mathrm{ro}} & =M e^{-\mu_{2} t} \\
V_{3 \mathrm{ro}} & =\frac{\left(C_{D}-\omega \epsilon\right)}{\mu_{2}} e^{-\mu_{2} t}\left[-e^{\mu_{2} t}+e^{\mu_{2} t_{2 r}}\right] .
\end{aligned}
$$

Since equations (4.9) and (4.10) satisfy the continuous condition at $t=t_{r}$, we obtain

$$
M=\frac{\left(C_{D}-\omega \epsilon\right)}{\mu_{2}}\left[-e^{\mu_{2} t_{r}}+e^{\mu_{2} t_{2 r}}\right]
$$

Assuming that one manufacturing cycle contains the retailer's cycle, $Z_{r}$, the cycle time is given by $t_{2 r}=\frac{T}{N Z_{r}}$ and $t_{2 r}=2 t_{r}$.

\section{Remanufacturing cycle}

Product collection occurs throughout the cycle during manufacturing and remanufacturing. The used and returned products are stored and returned to the manufacturer for remanufacturing. Considering the customer demand as $C_{D}$, and $\rho$ as the returned rate, the collected inventory at time $t$ is given by

$$
V_{C}(t)=\rho \sigma C_{D}
$$

The manufacturer utilizes this collected inventory to remanufacture products while recycling waste. Reproduction begins at time $t=0$ and continues until $t_{R}$. The inventory level reduces owing to the demand $\alpha_{1}+\alpha_{2} t$ and 
deterioration $\mu_{1}$. At $t_{R}$ sufficient inventory is accumulated, and the production is stopped. The inventory level continues to reduce owing to the deterioration $\mu_{1}$ and demand until time $T$, when the inventory becomes 0 .

$$
\frac{\mathrm{d} V_{R}}{\mathrm{~d} t}=P_{R}-\left(\alpha_{1}+\alpha_{2} t\right)-\mu_{1} V_{R}, \quad 0 \leq t \leq t_{R}
$$

with the boundary condition $V_{R}(0)=0$.

$$
\frac{\mathrm{d} V_{2 R}}{\mathrm{~d} t}=-\left(\alpha_{1}+\alpha_{2} t\right)-\mu_{1} V_{2 R}, \quad t_{R} \leq t \leq T
$$

with the boundary condition $V_{2 R}(T)=0$.

Then, the solutions of both equations are

$$
\begin{aligned}
V_{R} & =\frac{e^{-\mu_{1} t}}{\mu_{1}^{2}}\left[\left\{\left(P_{R}-\left(\alpha_{1}+\alpha_{2} t\right)\right) \mu_{1}+\alpha_{2}\right\} e^{\mu_{1} t}-\left\{\left(P_{R}-\alpha_{1}\right) \mu_{1}+\alpha_{2}\right\}\right] \\
V_{2 R} & =\frac{e^{-\mu_{1} t}}{\mu_{1}^{2}}\left[\left(\alpha_{2}-\left(\alpha_{1}+\alpha_{2} t\right) \mu_{1}\right) e^{\mu_{1} t}+\left(\left(\alpha_{1}+\alpha_{2} T\right) \mu_{1}-\alpha_{2}\right) e^{\mu_{1} T}\right] .
\end{aligned}
$$

\section{Manufacturing cost}

Setup cost: $S_{\mathrm{MC}}$.

Production cost:

$$
P_{\mathrm{MC}} \int_{0}^{t_{m}} e^{-\tau t} P_{M} \mathrm{~d} t=P_{\mathrm{MC}}\left(1-e^{-\tau t_{m}}\right) \frac{P_{M}}{\tau} .
$$

Holding cost:

$$
\begin{aligned}
H_{\mathrm{MC}} & {\left[\int_{0}^{t_{m}} e^{-\tau t} V_{m}(t) \mathrm{d} t+\int_{t_{m}}^{t_{2 m}} e^{-\tau t} V_{2 m}(t) \mathrm{d} t\right] } \\
= & H_{\mathrm{MC}} \frac{e^{-t_{M}\left(\tau+\mu_{1}\right)}}{\tau^{2} \mu_{1}^{2}\left(\tau+\mu_{1}\right)}\left[-\tau\left(P_{M}-\alpha_{1}\right) \mu_{1}\left\{\left(-1+e^{t_{m} \mu_{1}}\right) \tau-e^{t_{m} \mu_{1}}\left(-1+e^{\tau t_{m}}\right) \mu_{1}\right\}+\alpha_{2}\left\{-\left(-1+e^{t_{m} \mu_{1}}\right) \tau^{2}\right.\right. \\
& \left.\left.+e^{t_{m} \mu_{1}} \mu_{1}\left(-\left(-1+e^{\tau t_{m}}\right) \mu_{1}+\tau t_{m}\left(\tau+\mu_{1}\right)\right)\right\}\right]+\frac{1}{\mu_{1}^{2}} H_{\mathrm{MC}}\left[e ^ { - t _ { m } ( \tau + \mu _ { 1 } ) } \left\{\frac{e^{t_{2 m} \mu_{1}}\left(\alpha_{1} \mu_{1}+\alpha_{2}\left(-1+t_{2 m} \mu_{1}\right)\right)}{\tau+\mu_{1}}\right.\right. \\
& \left.-\frac{e^{t_{m} \mu_{1}}\left(\tau \alpha_{1} \mu_{1}+\alpha_{2}\left(-\tau+\left(1+\tau t_{m}\right) \mu_{1}\right)\right)}{\tau^{2}}\right\}+e^{-t_{2 m}\left(\tau+\mu_{1}\right)}\left\{-\frac{e^{t_{2 m} \mu_{1}}\left(\alpha_{1} \mu_{1}+\alpha_{2}\left(-1+t_{2 m} \mu_{1}\right)\right)}{\tau+\mu_{1}}\right. \\
& \left.\left.+\frac{e^{t_{2 m} \mu_{1}}\left(\tau \alpha_{1} \mu_{1}+\alpha_{2}\left(-\tau+\left(1+\tau t_{2 m}\right) \mu_{1}\right)\right)}{\tau^{2}}\right\}\right] .
\end{aligned}
$$

Deterioration cost:

$$
\begin{aligned}
D_{\mathrm{MC}} & {\left[\int_{0}^{t_{m}} \mu_{1} e^{-\tau t} V_{m}(t) \mathrm{d} t+\int_{t_{m}}^{t_{2 m}} \mu_{1} e^{-\tau t} V_{2 m}(t) \mathrm{d} t\right] } \\
= & \mu_{1} D_{\mathrm{MC}} \frac{e^{-t_{M}\left(\tau+\mu_{1}\right)}}{\tau^{2} \mu_{1}^{2}\left(\tau+\mu_{1}\right)}\left[-\tau\left(P_{M}-\alpha_{1}\right) \mu_{1}\left\{\left(-1+e^{t_{m} \mu_{1}}\right) \tau-e^{t_{m} \mu_{1}}\left(-1+e^{\tau t_{m}}\right) \mu_{1}\right\}+\alpha_{2}\left\{-\left(-1+e^{t_{m} \mu_{1}}\right) \tau^{2}\right.\right. \\
& \left.\left.+e^{t_{m} \mu_{1}} \mu_{1}\left(-\left(-1+e^{\tau t_{m}}\right) \mu_{1}+\tau t_{m}\left(\tau+\mu_{1}\right)\right)\right\}\right]+\frac{1}{\mu_{1}^{2}} \mu_{1} D_{\mathrm{MC}}\left[e ^ { - t _ { m } ( \tau + \mu _ { 1 } ) } \left\{\frac{e^{t_{2 m} \mu_{1}}\left(\alpha_{1} \mu_{1}+\alpha_{2}\left(-1+t_{2 m} \mu_{1}\right)\right)}{\tau+\mu_{1}}\right.\right. \\
& \left.-\frac{e^{t_{m} \mu_{1}}\left(\tau \alpha_{1} \mu_{1}+\alpha_{2}\left(-\tau+\left(1+\tau t_{m}\right) \mu_{1}\right)\right)}{\tau^{2}}\right\}+e^{-t_{2 m}\left(\tau+\mu_{1}\right)}\left\{-\frac{e^{t_{2 m} \mu_{1}}\left(\alpha_{1} \mu_{1}+\alpha_{2}\left(-1+t_{2 m} \mu_{1}\right)\right)}{\tau+\mu_{1}}\right. \\
& \left.\left.+\frac{e^{t_{2 m} \mu_{1}}\left(\tau \alpha_{1} \mu_{1}+\alpha_{2}\left(-\tau+\left(1+\tau t_{2 m}\right) \mu_{1}\right)\right)}{\tau^{2}}\right\}\right] .
\end{aligned}
$$




\section{Retailer cost}

Ordering cost: $O_{\mathrm{rC}}$.

Purchasing cost:

$$
P_{\mathrm{rC}}\left[V_{\mathrm{rr}}(0)+M\right]=\frac{P_{\mathrm{rC}}\left(C_{D}-\omega \epsilon\right)}{\mu_{2}}\left[-e^{\mu_{2} t_{r}}+e^{\mu_{2} t_{2 r}}\right] .
$$

Holding cost:

$$
\begin{aligned}
& {\left[H_{\mathrm{rrC}} \int_{0}^{t_{r}} e^{-\tau t} V_{\mathrm{rr}}(t) \mathrm{d} t+H_{\mathrm{roC}}\left\{\int_{0}^{t_{r}} e^{-\tau t} V_{2 \mathrm{ro}}(t) \mathrm{d} t+\int_{t_{r}}^{t_{2 r}} e^{-\tau t} V_{3 \mathrm{ro}}(t) \mathrm{d} t\right\}\right]} \\
& =\frac{H_{\mathrm{rrC}}\left(C_{D}-\omega \epsilon\right)}{\mu_{2}}\left(\frac{-1+e^{-\tau t_{r}}}{\tau}+\frac{1-e^{-t_{r}\left(\tau+\mu_{2}\right)}}{\tau+\mu_{2}}\right)+\frac{H_{\mathrm{roC}}}{\tau+\mu_{2}}\left(1-e^{-t_{r}\left(\tau+\mu_{2}\right)}\right) \frac{\left(C_{D}-\omega \epsilon\right)}{\mu_{2}}\left(-e^{\mu_{2} t_{r}}+e^{\mu_{2} t_{2 r}}\right) \\
& \quad+\frac{H_{\mathrm{roC}}\left(C_{D}-\omega \epsilon\right)}{\mu_{2}}\left(-\frac{e^{-\tau t_{r}}-e^{-\tau t_{2 r}}}{\tau}+\frac{e^{t_{2 r} \mu_{2}}\left(e^{-t_{r}\left(\tau+\mu_{2}\right)}-e^{-t_{2 r}\left(\tau+\mu_{2}\right)}\right)}{\tau+\mu_{2}}\right) .
\end{aligned}
$$

Deterioration cost:

$$
\begin{aligned}
\mu_{2} D_{\mathrm{rC}} & {\left[\int_{0}^{t_{r}} e^{-\tau t} V_{\mathrm{rr}}(t) \mathrm{d} t+\int_{0}^{t_{r}} e^{-\tau t} V_{2 \mathrm{ro}}(t) \mathrm{d} t+\int_{t_{r}}^{t_{2 r}} e^{-\tau t} V_{3 \mathrm{ro}}(t) \mathrm{d} t\right] } \\
= & \mu_{2} D_{\mathrm{rC}}\left[\frac{\left(C_{D}-\omega \epsilon\right)}{\mu_{2}}\left(\frac{-1+e^{-\tau t_{r}}}{\tau}+\frac{1-e^{-t_{r}\left(\tau+\mu_{2}\right)}}{\tau+\mu_{2}}\right)+\frac{1}{\tau+\mu_{2}}\left(1-e^{-t_{r}\left(\tau+\mu_{2}\right)}\right) \frac{\left(C_{D}-\omega \epsilon\right)}{\mu_{2}}\right. \\
& \left.\times\left(-e^{\mu_{2} t_{r}}+e^{\mu_{2} t_{2 r}}\right)+\frac{\left(C_{D}-\omega \epsilon\right)}{\mu_{2}}\left(-\frac{e^{-\tau t_{r}}-e^{-\tau t_{2 r}}}{\tau}+\frac{e^{t_{2 r} \mu_{2}}\left(e^{-t_{r}\left(\tau+\mu_{2}\right)}-e^{-t_{2 r}\left(\tau+\mu_{2}\right)}\right)}{\tau+\mu_{2}}\right)\right] .
\end{aligned}
$$

\section{Remanufacturing cost}

Setup cost: $S_{\mathrm{RC}}$.

Production cost:

$$
P_{\mathrm{RC}} \int_{0}^{t_{R}} e^{-\tau t} P_{R} \mathrm{~d} t=\frac{P_{\mathrm{RC}}}{\tau}\left(1-e^{-\tau t_{R}}\right) P_{R} .
$$

Holding cost:

$$
\begin{aligned}
H_{\mathrm{RC}} & {\left[\int_{0}^{t_{R}} e^{-\tau t} V_{R}(t) \mathrm{d} t+\int_{t_{R}}^{T} e^{-\tau t} V_{2 R}(t) \mathrm{d} t\right] } \\
= & H_{\mathrm{RC}} \frac{e^{-t_{R}\left(\tau+\mu_{1}\right)}}{\tau^{2} \mu_{1}^{2}\left(\tau+\mu_{1}\right)}\left[-\tau\left(P_{R}-\alpha_{1}\right) \mu_{1}\left\{\left(-1+e^{t_{R} \mu_{1}}\right) \tau-e^{t_{R} \mu_{1}}\left(-1+e^{\tau t_{R}}\right) \mu_{1}\right\}+\alpha_{2}\left\{-\left(-1+e^{t_{R} \mu_{1}}\right) \tau^{2}\right.\right. \\
& \left.\left.+e^{t_{R} \mu_{1}} \mu_{1}\left(-\left(-1+e^{\tau t_{R}}\right) \mu_{1}+\tau t_{R}\left(\tau+\mu_{1}\right)\right)\right\}\right]+\frac{1}{\mu_{1}^{2}} H_{\mathrm{RC}}\left[e ^ { - t _ { R } ( \tau + \mu _ { 1 } ) } \left\{\frac{e^{T \mu_{1}}\left(\alpha_{1} \mu_{1}+\alpha_{2}\left(-1+T \mu_{1}\right)\right)}{\tau+\mu_{1}}\right.\right. \\
& \left.-\frac{e^{t_{R} \mu_{1}}\left(\tau \alpha_{1} \mu_{1}+\alpha_{2}\left(-\tau+\left(1+\tau t_{R}\right) \mu_{1}\right)\right)}{\tau^{2}}\right\}+e^{-T\left(\tau+\mu_{1}\right)}\left\{-\frac{e^{T \mu_{1}}\left(\alpha_{1} \mu_{1}+\alpha_{2}\left(-1+T \mu_{1}\right)\right)}{\tau+\mu_{1}}\right. \\
& \left.\left.+\frac{e^{T \mu_{1}}\left(\tau \alpha_{1} \mu_{1}+\alpha_{2}\left(-\tau+(1+\tau T) \mu_{1}\right)\right)}{\tau^{2}}\right\}\right] .
\end{aligned}
$$

Deterioration cost:

$D_{\mathrm{RC}}\left[\int_{0}^{t_{R}} \mu_{1} e^{-\tau t} V_{R}(t) \mathrm{d} t+\int_{t_{R}}^{T} \mu_{1} e^{-\tau t} V_{2 R}(t) \mathrm{d} t\right]$ 


$$
\begin{aligned}
= & \mu_{1} D_{\mathrm{RC}} \frac{e^{-t_{R}\left(\tau+\mu_{1}\right)}}{\tau^{2} \mu_{1}^{2}\left(\tau+\mu_{1}\right)}\left[-\tau\left(P_{R}-\alpha_{1}\right) \mu_{1}\left\{\left(-1+e^{t_{R} \mu_{1}}\right) \tau-e^{t_{R} \mu_{1}}\left(-1+e^{\tau t_{R}}\right) \mu_{1}\right\}+\alpha_{2}\left\{-\left(-1+e^{t_{R} \mu_{1}}\right) \tau^{2}\right.\right. \\
& \left.\left.+e^{t_{R} \mu_{1}} \mu_{1}\left(-\left(-1+e^{\tau t_{R}}\right) \mu_{1}+\tau t_{R}\left(\tau+\mu_{1}\right)\right)\right\}\right]+\frac{1}{\mu_{1}^{2}} \mu_{1} D_{\mathrm{RC}}\left[e ^ { - t _ { R } ( \tau + \mu _ { 1 } ) } \left\{\frac{e^{T \mu_{1}}\left(\alpha_{1} \mu_{1}+\alpha_{2}\left(-1+T \mu_{1}\right)\right)}{\tau+\mu_{1}}\right.\right. \\
& \left.-\frac{e^{t_{R} \mu_{1}}\left(\tau \alpha_{1} \mu_{1}+\alpha_{2}\left(-\tau+\left(1+\tau t_{R}\right) \mu_{1}\right)\right)}{\tau^{2}}\right\}+e^{-T\left(\tau+\mu_{1}\right)}\left\{-\frac{e^{T \mu_{1}}\left(\alpha_{1} \mu_{1}+\alpha_{2}\left(-1+T \mu_{1}\right)\right)}{\tau+\mu_{1}}\right. \\
& \left.\left.+\frac{e^{T \mu_{1}}\left(\tau \alpha_{1} \mu_{1}+\alpha_{2}\left(-\tau+(1+\tau T) \mu_{1}\right)\right)}{\tau^{2}}\right\}\right] .
\end{aligned}
$$

Collection cost:

$$
C_{\mathrm{RC}} \int_{0}^{T} e^{-\tau t} V_{C}(t) \mathrm{d} t=\frac{C_{\mathrm{RC}}}{\tau}\left(1-e^{-T \tau}\right) \rho \sigma C_{D}
$$

Total cost:

$$
\begin{aligned}
& \mathrm{TS}_{C}=\frac{\mathrm{N}}{\mathrm{T}}\left[S_{\mathrm{MC}}+P_{\mathrm{MC}}\left(1-e^{-\tau t_{m}}\right) \frac{P_{M}}{\tau}+H_{\mathrm{MC}} \frac{e^{-t_{M}\left(\tau+\mu_{1}\right)}}{\tau^{2} \mu_{1}^{2}\left(\tau+\mu_{1}\right)}\left[-\tau\left(P_{M}-\alpha_{1}\right) \mu_{1}\left\{\left(-1+e^{t_{m} \mu_{1}}\right) \tau\right.\right.\right. \\
& \left.\left.-e^{t_{m} \mu_{1}}\left(-1+e^{\tau t_{m}}\right) \mu_{1}\right\}+\alpha_{2}\left\{-\left(-1+e^{t_{m} \mu_{1}}\right) \tau^{2}+e^{t_{m} \mu_{1}} \mu_{1}\left(-\left(-1+e^{\tau t_{m}}\right) \mu_{1}+\tau t_{m}\left(\tau+\mu_{1}\right)\right)\right\}\right] \\
& +\frac{1}{\mu_{1}^{2}} H_{\mathrm{MC}}\left[e^{-t_{m}\left(\tau+\mu_{1}\right)}\left\{\frac{e^{t_{2 m} \mu_{1}}\left(\alpha_{1} \mu_{1}+\alpha_{2}\left(-1+t_{2 m} \mu_{1}\right)\right)}{\tau+\mu_{1}}-\frac{e^{t_{m} \mu_{1}}\left(\tau \alpha_{1} \mu_{1}+\alpha_{2}\left(-\tau+\left(1+\tau t_{m}\right) \mu_{1}\right)\right)}{\tau^{2}}\right\}\right. \\
& \left.+e^{-t_{2 m}\left(\tau+\mu_{1}\right)}\left\{-\frac{e^{t_{2 m} \mu_{1}}\left(\alpha_{1} \mu_{1}+\alpha_{2}\left(-1+t_{2 m} \mu_{1}\right)\right)}{\tau+\mu_{1}}+\frac{e^{t_{2 m} \mu_{1}}\left(\tau \alpha_{1} \mu_{1}+\alpha_{2}\left(-\tau+\left(1+\tau t_{2 m}\right) \mu_{1}\right)\right)}{\tau^{2}}\right\}\right] \\
& +\mu_{1} D_{\mathrm{MC}} \frac{e^{-t_{M}\left(\tau+\mu_{1}\right)}}{\tau^{2} \mu_{1}^{2}\left(\tau+\mu_{1}\right)}\left[-\tau\left(P_{M}-\alpha_{1}\right) \mu_{1}\left\{\left(-1+e^{t_{m} \mu_{1}}\right) \tau-e^{t_{m} \mu_{1}}\left(-1+e^{\tau t_{m}}\right) \mu_{1}\right\}\right. \\
& \left.+\alpha_{2}\left\{-\left(-1+e^{t_{m} \mu_{1}}\right) \tau^{2}+e^{t_{m} \mu_{1}} \mu_{1}\left(-\left(-1+e^{\tau t_{m}}\right) \mu_{1}+\tau t_{m}\left(\tau+\mu_{1}\right)\right)\right\}\right]+\frac{1}{\mu_{1}^{2}} \mu_{1} D_{\mathrm{MC}} \\
& \times\left[e^{-t_{m}\left(\tau+\mu_{1}\right)}\left\{\frac{e^{t_{2 m} \mu_{1}}\left(\alpha_{1} \mu_{1}+\alpha_{2}\left(-1+t_{2 m} \mu_{1}\right)\right)}{\tau+\mu_{1}}-\frac{e^{t_{m} \mu_{1}}\left(\tau \alpha_{1} \mu_{1}+\alpha_{2}\left(-\tau+\left(1+\tau t_{m}\right) \mu_{1}\right)\right)}{\tau^{2}}\right\}\right. \\
& \left.\left.+e^{-t_{2 m}\left(\tau+\mu_{1}\right)}\left\{-\frac{e^{t_{2 m} \mu_{1}}\left(\alpha_{1} \mu_{1}+\alpha_{2}\left(-1+t_{2 m} \mu_{1}\right)\right)}{\tau+\mu_{1}}+\frac{e^{t_{2 m} \mu_{1}}\left(\tau \alpha_{1} \mu_{1}+\alpha_{2}\left(-\tau+\left(1+\tau t_{2 m}\right) \mu_{1}\right)\right)}{\tau^{2}}\right\}\right]\right] \\
& +\frac{\mathrm{NZ}}{\mathrm{T}}\left[O_{\mathrm{rC}}+\frac{P_{\mathrm{rC}}\left(C_{D}-\omega \epsilon\right)}{\mu_{2}}\left[-e^{\mu_{2} t_{r}}+e^{\mu_{2} t_{2 r}}\right]+\frac{H_{\mathrm{rrC}}\left(C_{D}-\omega \epsilon\right)}{\mu_{2}}\left(\frac{-1+e^{-\tau t_{r}}}{\tau}+\frac{1-e^{-t_{r}\left(\tau+\mu_{2}\right)}}{\tau+\mu_{2}}\right)\right. \\
& +\frac{H_{\mathrm{roC}}}{\tau+\mu_{2}}\left(1-e^{-t_{r}\left(\tau+\mu_{2}\right)}\right) \frac{\left(C_{D}-\omega \epsilon\right)}{\mu_{2}}\left(-e^{\mu_{2} t_{r}}+e^{\mu_{2} t_{2 r}}\right)+\frac{H_{\mathrm{roC}}\left(C_{D}-\omega \epsilon\right)}{\mu_{2}}\left(-\frac{e^{-\tau t_{r}}-e^{-\tau t_{2 r}}}{\tau}\right. \\
& \left.+\frac{e^{t_{2 r} \mu_{2}}\left(e^{-t_{r}\left(\tau+\mu_{2}\right)}-e^{-t_{2 r}\left(\tau+\mu_{2}\right)}\right)}{\tau+\mu_{2}}\right)+\mu_{2} D_{\mathrm{rC}}\left[\frac{\left(C_{D}-\omega \epsilon\right)}{\mu_{2}}\left(\frac{-1+e^{-\tau t_{r}}}{\tau}+\frac{1-e^{-t_{r}\left(\tau+\mu_{2}\right)}}{\tau+\mu_{2}}\right)\right. \\
& +\frac{1}{\tau+\mu_{2}}\left(1-e^{-t_{r}\left(\tau+\mu_{2}\right)}\right) \frac{\left(C_{D}-\omega \epsilon\right)}{\mu_{2}}\left(-e^{\mu_{2} t_{r}}+e^{\mu_{2} t_{2 r}}\right)+\frac{\left(C_{D}-\omega \epsilon\right)}{\mu_{2}}\left(-\frac{e^{-\tau t_{r}}-e^{-\tau t_{2 r}}}{\tau}\right. \\
& \left.\left.\left.+\frac{e^{t_{2 r} \mu_{2}}\left(e^{-t_{r}\left(\tau+\mu_{2}\right)}-e^{-t_{2 r}\left(\tau+\mu_{2}\right)}\right)}{\tau+\mu_{2}}\right)\right]\right]+\frac{1}{\mathrm{~T}}\left[S_{\mathrm{RC}}+\frac{P_{\mathrm{RC}}}{\tau}\left(1-e^{-\tau t_{R}}\right) P_{R}+H_{\mathrm{RC}} \frac{e^{-t_{R}\left(\tau+\mu_{1}\right)}}{\tau^{2} \mu_{1}^{2}\left(\tau+\mu_{1}\right)}\right. \\
& \times\left[-\tau\left(P_{R}-\alpha_{1}\right) \mu_{1}\left\{\left(-1+e^{t_{R} \mu_{1}}\right) \tau-e^{t_{R} \mu_{1}}\left(-1+e^{\tau t_{R}}\right) \mu_{1}\right\}+\alpha_{2}\left\{-\left(-1+e^{t_{R} \mu_{1}}\right)+e^{t_{R} \mu_{1}} \mu_{1} \tau^{2}\right.\right. \\
& \left.\left.\times\left(-\left(-1+e^{\tau t_{R}}\right) \mu_{1}+\tau t_{R}\left(\tau+\mu_{1}\right)\right)\right\}\right]+\frac{1}{\mu_{1}^{2}} H_{\mathrm{RC}}\left[e ^ { - t _ { R } ( \tau + \mu _ { 1 } ) } \left\{\frac{e^{T \mu_{1}}\left(\alpha_{1} \mu_{1}+\alpha_{2}\left(-1+T \mu_{1}\right)\right)}{\tau+\mu_{1}}\right.\right.
\end{aligned}
$$




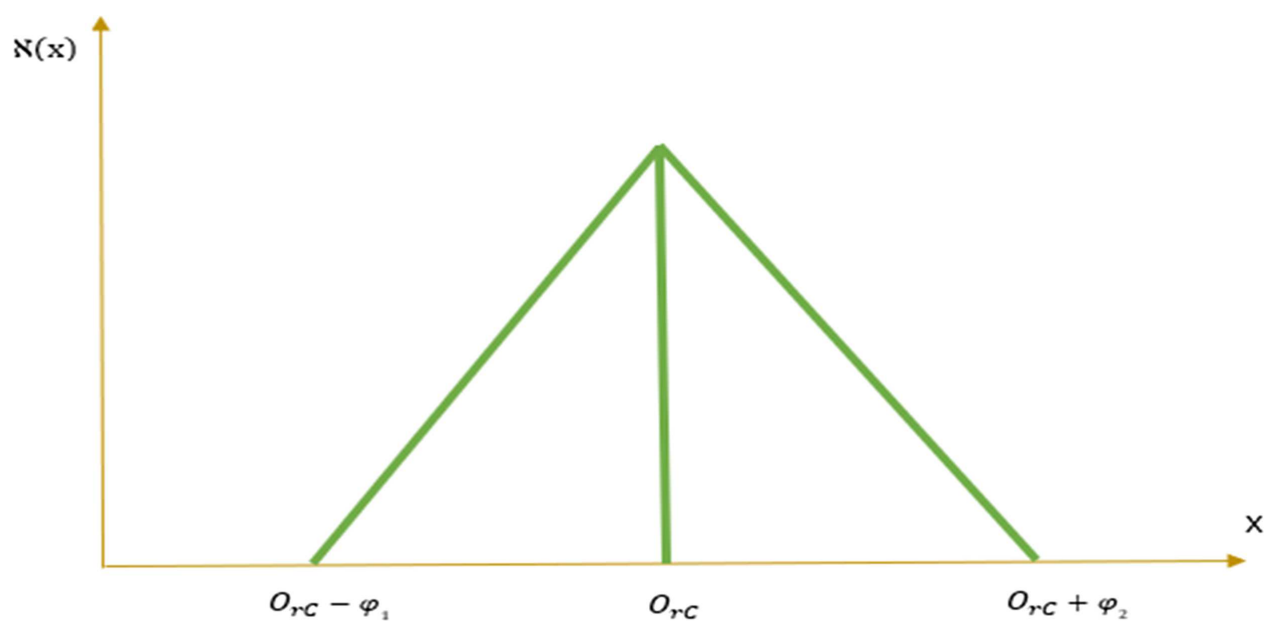

Figure 3. Triangular fuzzy number $O_{\mathrm{rC}}$.

$$
\begin{aligned}
& \left.-\frac{e^{t_{R} \mu_{1}}\left(\tau \alpha_{1} \mu_{1}+\alpha_{2}\left(-\tau+\left(1+\tau t_{R}\right) \mu_{1}\right)\right)}{\tau^{2}}\right\}+e^{-T\left(\tau+\mu_{1}\right)}\left\{-\frac{e^{T \mu_{1}}\left(\alpha_{1} \mu_{1}+\alpha_{2}\left(-1+T \mu_{1}\right)\right)}{\tau+\mu_{1}}\right. \\
& \left.\left.+\frac{e^{T \mu_{1}}\left(\tau \alpha_{1} \mu_{1}+\alpha_{2}\left(-\tau+(1+\tau T) \mu_{1}\right)\right)}{\tau^{2}}\right\}\right]+\mu_{1} D_{\mathrm{RC}} \frac{e^{-t_{R}\left(\tau+\mu_{1}\right)}}{\tau^{2} \mu_{1}^{2}\left(\tau+\mu_{1}\right)}\left[-\tau\left(P_{R}-\alpha_{1}\right) \mu_{1}\left\{\left(-1+e^{t_{R} \mu_{1}}\right) \tau\right.\right. \\
& \left.\left.-e^{t_{R} \mu_{1}}\left(-1+e^{\tau t_{R}}\right) \mu_{1}\right\}+\alpha_{2}\left\{-\left(-1+e^{t_{R} \mu_{1}}\right) \tau^{2}+e^{t_{R} \mu_{1}} \mu_{1}\left(-\left(-1+e^{\tau t_{R}}\right) \mu_{1}+\tau t_{R}\left(\tau+\mu_{1}\right)\right)\right\}\right] \\
& +\frac{1}{\mu_{1}^{2}} \mu_{1} D_{\mathrm{RC}}\left[e^{-t_{R}\left(\tau+\mu_{1}\right)}\left\{\frac{e^{T \mu_{1}}\left(\alpha_{1} \mu_{1}+\alpha_{2}\left(-1+T \mu_{1}\right)\right)}{\tau+\mu_{1}}-\frac{e^{t_{R} \mu_{1}}\left(\tau \alpha_{1} \mu_{1}+\alpha_{2}\left(-\tau+\left(1+\tau t_{R}\right) \mu_{1}\right)\right)}{\tau^{2}}\right\}\right. \\
& \left.+e^{-T\left(\tau+\mu_{1}\right)}\left\{-\frac{e^{T \mu_{1}}\left(\alpha_{1} \mu_{1}+\alpha_{2}\left(-1+T \mu_{1}\right)\right)}{\tau+\mu_{1}}+\frac{e^{T \mu_{1}}\left(\tau \alpha_{1} \mu_{1}+\alpha_{2}\left(-\tau+(1+\tau T) \mu_{1}\right)\right)}{\tau^{2}}\right\}\right] \\
& \left.+\frac{C_{\mathrm{RC}}}{\tau}\left(1-e^{-T \tau}\right) \rho \sigma C_{D}\right] .
\end{aligned}
$$

\section{Fuzzy model}

This study considered $O_{\mathrm{rC}}, P_{\mathrm{MC}}, P_{\mathrm{RC}}$ and $\omega$ as triangular fuzzy numbers, that is, $\widetilde{O_{\mathrm{rC}}}, \widetilde{P_{\mathrm{MC}}}, \widetilde{P_{\mathrm{RC}}}$, and $\widetilde{\omega}$. This study assumed that the parameters $\widetilde{O_{\mathrm{rC}}}, \widetilde{P_{\mathrm{MC}}}, \widetilde{P_{\mathrm{RC}}}$, and $\widetilde{\omega}$ may change within certain limits.

The membership order cost fuzzy number (see Fig. 3) can be defined as

$$
\aleph_{\widetilde{O_{\mathrm{rC}}}}(x)= \begin{cases}\frac{x-\left(O_{\mathrm{rC}}-\varphi_{1}\right)}{\varphi_{1}} & \text { if } O_{\mathrm{rC}}-\varphi_{1}<z<O_{\mathrm{rC}} \\ \frac{\left(O_{\mathrm{rC}}+\varphi_{2}\right)-x}{\varphi_{2}} & \text { if } O_{\mathrm{rC}}<z<O_{\mathrm{rC}}+\varphi_{2} \\ 0 & \text { if otherwise, }\end{cases}
$$

and can be expressed as

$$
\widetilde{O_{\mathrm{rC}}}=\left(O_{\mathrm{rC}}-\varphi_{1}, O_{\mathrm{rC}}, O_{\mathrm{rC}}+\varphi_{2}\right), \text { where } 0<\varphi_{1}<O_{\mathrm{rC}} \quad \text { and } \varphi_{1} \varphi_{2}>0 .
$$

Similarly, other fuzzy numbers can be expressed as

$$
\begin{aligned}
\widetilde{P_{\mathrm{MC}}} & =\left(P_{\mathrm{MC}}-\varphi_{3}, P_{\mathrm{MC}}, P_{\mathrm{MC}}+\varphi_{4}\right), \text { where } 0<\varphi_{3}<P_{\mathrm{MC}} \text { and } \varphi_{3} \varphi_{4}>0 \\
\widetilde{P_{\mathrm{RC}}} & =\left(P_{\mathrm{RC}}-\varphi_{5}, P_{\mathrm{RC}}, P_{\mathrm{RC}}+\varphi_{6}\right), \text { where } 0<\varphi_{5}<P_{\mathrm{RC}} \text { and } \varphi_{5} \varphi_{6}>0
\end{aligned}
$$




$$
\widetilde{\omega}=\left(\omega-\varphi_{7}, \omega, \omega+\varphi_{8}\right) \text {, where } 0<\varphi_{7}<\omega \text { and } \varphi_{7} \varphi_{8}>0 .
$$

Using the signed distance method (SDM), this study obtains

$$
\begin{aligned}
\widetilde{O_{\mathrm{rC}}} & =O_{\mathrm{rC}}+\frac{1}{4}\left(\varphi_{2}-\varphi_{1}\right) \\
\widetilde{P_{\mathrm{MC}}} & =A_{s}+\frac{1}{4}\left(\varphi_{4}-\varphi_{3}\right) \\
\widetilde{P_{\mathrm{RC}}} & =P_{\mathrm{RC}}+\frac{1}{4}\left(\varphi_{6}-\varphi_{5}\right) \\
\widetilde{\omega} & =\omega+\frac{1}{4}\left(\varphi_{8}-\varphi_{7}\right) .
\end{aligned}
$$

Hence, the defuzzification of the total profit using SDM is

$$
\begin{aligned}
& \widetilde{\mathrm{TS}_{C}^{\prime}}=\frac{1}{4}\left[\widetilde{\mathrm{TS}_{C 1}}+\widetilde{2 \mathrm{TS}_{C 2}}+\widetilde{\mathrm{TS}_{C 3}}\right] \\
& \mathrm{TS}_{C 1}=\frac{\mathrm{N}}{\mathrm{T}}\left[S_{\mathrm{MC}}+\left(P_{\mathrm{MC}}-\varphi_{3}\right)\left(1-e^{-\tau t_{m}}\right) \frac{P_{M}}{\tau}+H_{\mathrm{MC}} \frac{e^{-t_{M}\left(\tau+\mu_{1}\right)}}{\tau^{2} \mu_{1}^{2}\left(\tau+\mu_{1}\right)}\left[-\tau\left(P_{M}-\alpha_{1}\right) \mu_{1}\left\{\left(-1+e^{t_{m} \mu_{1}}\right) \tau\right.\right.\right. \\
& \left.\left.-e^{t_{m} \mu_{1}}\left(-1+e^{\tau t_{m}}\right) \mu_{1}\right\}+\alpha_{2}\left\{-\left(-1+e^{t_{m} \mu_{1}}\right) \tau^{2}+e^{t_{m} \mu_{1}} \mu_{1}\left(-\left(-1+e^{\tau t_{m}}\right) \mu_{1}+\tau t_{m}\left(\tau+\mu_{1}\right)\right)\right\}\right] \\
& +\frac{1}{\mu_{1}^{2}} H_{\mathrm{MC}}\left[e^{-t_{m}\left(\tau+\mu_{1}\right)}\left\{\frac{e^{t_{2 m} \mu_{1}}\left(\alpha_{1} \mu_{1}+\alpha_{2}\left(-1+t_{2 m} \mu_{1}\right)\right)}{\tau+\mu_{1}}-\frac{e^{t_{m} \mu_{1}}\left(\tau \alpha_{1} \mu_{1}+\alpha_{2}\left(-\tau+\left(1+\tau t_{m}\right) \mu_{1}\right)\right)}{\tau^{2}}\right\}\right. \\
& \left.+e^{-t_{2 m}\left(\tau+\mu_{1}\right)}\left\{-\frac{e^{t_{2 m} \mu_{1}}\left(\alpha_{1} \mu_{1}+\alpha_{2}\left(-1+t_{2 m} \mu_{1}\right)\right)}{\tau+\mu_{1}}+\frac{e^{t_{2 m} \mu_{1}}\left(\tau \alpha_{1} \mu_{1}+\alpha_{2}\left(-\tau+\left(1+\tau t_{2 m}\right) \mu_{1}\right)\right)}{\tau^{2}}\right\}\right] \\
& +\mu_{1} D_{\mathrm{MC}} \frac{e^{-t_{M}\left(\tau+\mu_{1}\right)}}{\tau^{2} \mu_{1}^{2}\left(\tau+\mu_{1}\right)}\left[-\tau\left(P_{M}-\alpha_{1}\right) \mu_{1}\left\{\left(-1+e^{t_{m} \mu_{1}}\right) \tau-e^{t_{m} \mu_{1}}\left(-1+e^{\tau t_{m}}\right) \mu_{1}\right\}\right. \\
& \left.+\alpha_{2}\left\{-\left(-1+e^{t_{m} \mu_{1}}\right) \tau^{2}+e^{t_{m} \mu_{1}} \mu_{1}\left(-\left(-1+e^{\tau t_{m}}\right) \mu_{1}+\tau t_{m}\left(\tau+\mu_{1}\right)\right)\right\}\right]+\frac{1}{\mu_{1}^{2}} \mu_{1} D_{\mathrm{MC}} \\
& \times\left[e^{-t_{m}\left(\tau+\mu_{1}\right)}\left\{\frac{e^{t_{2 m} \mu_{1}}\left(\alpha_{1} \mu_{1}+\alpha_{2}\left(-1+t_{2 m} \mu_{1}\right)\right)}{\tau+\mu_{1}}-\frac{e^{t_{m} \mu_{1}}\left(\tau \alpha_{1} \mu_{1}+\alpha_{2}\left(-\tau+\left(1+\tau t_{m}\right) \mu_{1}\right)\right)}{\tau^{2}}\right\}\right. \\
& \left.\left.+e^{-t_{2 m}\left(\tau+\mu_{1}\right)}\left\{-\frac{e^{t_{2 m} \mu_{1}}\left(\alpha_{1} \mu_{1}+\alpha_{2}\left(-1+t_{2 m} \mu_{1}\right)\right)}{\tau+\mu_{1}}+\frac{e^{t_{2 m} \mu_{1}}\left(\tau \alpha_{1} \mu_{1}+\alpha_{2}\left(-\tau+\left(1+\tau t_{2 m}\right) \mu_{1}\right)\right)}{\tau^{2}}\right\}\right]\right] \\
& +\frac{\mathrm{NZ}}{\mathrm{T}}\left[\left(O_{\mathrm{rC}}-\varphi_{1}\right)+\frac{P_{\mathrm{rC}}\left(C_{D}-\left(\omega-\varphi_{7}\right) \epsilon\right)}{\mu_{2}}\left[-e^{\mu_{2} t_{1 r}}+e^{\mu_{2} t_{2 r}}\right]+\frac{H_{\mathrm{rrC}}\left(C_{D}-\left(\omega-\varphi_{7}\right) \epsilon\right)}{\mu_{2}}\left(\frac{-1+e^{-\tau t_{r}}}{\tau}\right.\right. \\
& \left.+\frac{1-e^{-t_{r}\left(\tau+\mu_{2}\right)}}{\tau+\mu_{2}}\right)+\frac{H_{\mathrm{roC}}}{\tau+\mu_{2}}\left(1-e^{-t_{r}\left(\tau+\mu_{2}\right)}\right) \frac{\left(C_{D}-\left(\omega-\varphi_{7}\right) \epsilon\right)}{\mu_{2}}\left(-e^{\mu_{2} t_{1 r}}+e^{\mu_{2} t_{2 r}}\right) \\
& +\frac{H_{\mathrm{roC}}\left(C_{D}-\left(\omega-\varphi_{7}\right) \epsilon\right)}{\mu_{2}}\left(-\frac{e^{-\tau t_{r}}-e^{-\tau t_{2 r}}}{\tau}+\frac{e^{t_{2 r} \mu_{2}}\left(e^{-t_{r}\left(\tau+\mu_{2}\right)}-e^{-t_{2 r}\left(\tau+\mu_{2}\right)}\right)}{\tau+\mu_{2}}\right)+\mu_{2} D_{\mathrm{rC}} \\
& \times\left[\frac{\left(C_{D}-\left(\omega-\varphi_{7}\right) \epsilon\right)}{\mu_{2}}\left(\frac{-1+e^{-\tau t_{r}}}{\tau}+\frac{1-e^{-t_{r}\left(\tau+\mu_{2}\right)}}{\tau+\mu_{2}}\right)+\frac{1}{\tau+\mu_{2}}\left(1-e^{-t_{r}\left(\tau+\mu_{2}\right)}\right) \frac{\left(C_{D}-\left(\omega-\varphi_{7}\right) \epsilon\right)}{\mu_{2}}\right. \\
& \left.\left.\times\left(-e^{\mu_{2} t_{1 r}}+e^{\mu_{2} t_{2 r}}\right)+\frac{\left(C_{D}-\left(\omega-\varphi_{7}\right) \epsilon\right)}{\mu_{2}}\left(-\frac{e^{-\tau t_{r}}-e^{-\tau t_{2 r}}}{\tau}+\frac{e^{t_{2 r} \mu_{2}}\left(e^{-t_{r}\left(\tau+\mu_{2}\right)}-e^{-t_{2 r}\left(\tau+\mu_{2}\right)}\right)}{\tau+\mu_{2}}\right)\right]\right] \\
& +\frac{1}{\mathrm{~T}}\left[S_{\mathrm{RC}}+\frac{\left(P_{\mathrm{RC}}-\varphi_{5}\right)}{\tau}\left(1-e^{-\tau t_{R}}\right) P_{R}+H_{\mathrm{RC}} \frac{e^{-t_{R}\left(\tau+\mu_{1}\right)}}{\tau^{2} \mu_{1}^{2}\left(\tau+\mu_{1}\right)}\left[-\tau\left(P_{R}-\alpha_{1}\right) \mu_{1}\left\{\left(-1+e^{t_{R} \mu_{1}}\right) \tau\right.\right.\right. \\
& \left.\left.-e^{t_{R} \mu_{1}}\left(-1+e^{\tau t_{R}}\right) \mu_{1}\right\}+\alpha_{2}\left\{-\left(-1+e^{t_{R} \mu_{1}}\right) \tau^{2}+e^{t_{R} \mu_{1}} \mu_{1}\left(-\left(-1+e^{\tau t_{R}}\right) \mu_{1}+\tau t_{R}\left(\tau+\mu_{1}\right)\right)\right\}\right]
\end{aligned}
$$




$$
\begin{aligned}
& +\frac{1}{\mu_{1}^{2}} H_{\mathrm{RC}}\left[e^{-t_{R}\left(\tau+\mu_{1}\right)}\left\{\frac{e^{T \mu_{1}}\left(\alpha_{1} \mu_{1}+\alpha_{2}\left(-1+T \mu_{1}\right)\right)}{\tau+\mu_{1}}-\frac{e^{t_{R} \mu_{1}}\left(\tau \alpha_{1} \mu_{1}+\alpha_{2}\left(-\tau+\left(1+\tau t_{R}\right) \mu_{1}\right)\right)}{\tau^{2}}\right\}\right. \\
& \left.+e^{-T\left(\tau+\mu_{1}\right)}\left\{-\frac{e^{T \mu_{1}}\left(\alpha_{1} \mu_{1}+\alpha_{2}\left(-1+T \mu_{1}\right)\right)}{\tau+\mu_{1}}+\frac{e^{T \mu_{1}}\left(\tau \alpha_{1} \mu_{1}+\alpha_{2}\left(-\tau+(1+\tau T) \mu_{1}\right)\right)}{\tau^{2}}\right\}\right] \\
& +\mu_{1} D_{\mathrm{RC}} \frac{e^{-t_{R}\left(\tau+\mu_{1}\right)}}{\tau^{2} \mu_{1}^{2}\left(\tau+\mu_{1}\right)}\left[-\tau\left(P_{R}-\alpha_{1}\right) \mu_{1}\left\{\left(-1+e^{t_{R} \mu_{1}}\right) \tau-e^{t_{R} \mu_{1}}\left(-1+e^{\tau t_{R}}\right) \mu_{1}\right\}\right. \\
& \left.+\alpha_{2}\left\{-\left(-1+e^{t_{R} \mu_{1}}\right) \tau^{2}+e^{t_{R} \mu_{1}} \mu_{1}\left(-\left(-1+e^{\tau t_{R}}\right) \mu_{1}+\tau t_{R}\left(\tau+\mu_{1}\right)\right)\right\}\right]+\frac{1}{\mu_{1}^{2}} \mu_{1} D_{\mathrm{RC}}\left[e^{-t_{R}\left(\tau+\mu_{1}\right)}\right. \\
& \times\left\{\frac{e^{T \mu_{1}}\left(\alpha_{1} \mu_{1}+\alpha_{2}\left(-1+T \mu_{1}\right)\right)}{\tau+\mu_{1}}-\frac{e^{t_{R} \mu_{1}}\left(\tau \alpha_{1} \mu_{1}+\alpha_{2}\left(-\tau+\left(1+\tau t_{R}\right) \mu_{1}\right)\right)}{\tau^{2}}\right\}+e^{-T\left(\tau+\mu_{1}\right)} \\
& \left.\left.\times\left\{-\frac{e^{T \mu_{1}}\left(\alpha_{1} \mu_{1}+\alpha_{2}\left(-1+T \mu_{1}\right)\right)}{\tau+\mu_{1}}+\frac{e^{T \mu_{1}}\left(\tau \alpha_{1} \mu_{1}+\alpha_{2}\left(-\tau+(1+\tau T) \mu_{1}\right)\right)}{\tau^{2}}\right\}\right]+\frac{C_{\mathrm{RC}}}{\tau}\left(1-e^{-T \tau}\right) \rho \sigma C_{D}\right] \\
& \widetilde{\mathrm{TS}_{C 2}}=\frac{\mathrm{N}}{\mathrm{T}}\left[S_{\mathrm{MC}}+P_{\mathrm{MC}}\left(1-e^{-\tau t_{m}}\right) \frac{P_{M}}{\tau}+H_{\mathrm{MC}} \frac{e^{-t_{M}\left(\tau+\mu_{1}\right)}}{\tau^{2} \mu_{1}^{2}\left(\tau+\mu_{1}\right)}\left[-\tau\left(P_{M}-\alpha_{1}\right) \mu_{1}\left\{\left(-1+e^{t_{m} \mu_{1}}\right) \tau\right.\right.\right. \\
& \left.\left.-e^{t_{m} \mu_{1}}\left(-1+e^{\tau t_{m}}\right) \mu_{1}\right\}+\alpha_{2}\left\{-\left(-1+e^{t_{m} \mu_{1}}\right) \tau^{2}+e^{t_{m} \mu_{1}} \mu_{1}\left(-\left(-1+e^{\tau t_{m}}\right) \mu_{1}+\tau t_{m}\left(\tau+\mu_{1}\right)\right)\right\}\right] \\
& +\frac{1}{\mu_{1}^{2}} H_{\mathrm{MC}}\left[e^{-t_{m}\left(\tau+\mu_{1}\right)}\left\{\frac{e^{t_{2 m} \mu_{1}}\left(\alpha_{1} \mu_{1}+\alpha_{2}\left(-1+t_{2 m} \mu_{1}\right)\right)}{\tau+\mu_{1}}-\frac{e^{t_{m} \mu_{1}}\left(\tau \alpha_{1} \mu_{1}+\alpha_{2}\left(-\tau+\left(1+\tau t_{m}\right) \mu_{1}\right)\right)}{\tau^{2}}\right\}\right. \\
& \left.+e^{-t_{2 m}\left(\tau+\mu_{1}\right)}\left\{-\frac{e^{t_{2 m} \mu_{1}}\left(\alpha_{1} \mu_{1}+\alpha_{2}\left(-1+t_{2 m} \mu_{1}\right)\right)}{\tau+\mu_{1}}+\frac{e^{t_{2 m} \mu_{1}}\left(\tau \alpha_{1} \mu_{1}+\alpha_{2}\left(-\tau+\left(1+\tau t_{2 m}\right) \mu_{1}\right)\right)}{\tau^{2}}\right\}\right] \\
& +\mu_{1} D_{\mathrm{MC}} \frac{e^{-t_{M}\left(\tau+\mu_{1}\right)}}{\tau^{2} \mu_{1}^{2}\left(\tau+\mu_{1}\right)}\left[-\tau\left(P_{M}-\alpha_{1}\right) \mu_{1}\left\{\left(-1+e^{t_{m} \mu_{1}}\right) \tau-e^{t_{m} \mu_{1}}\left(-1+e^{\tau t_{m}}\right) \mu_{1}\right\}\right. \\
& \left.+\alpha_{2}\left\{-\left(-1+e^{t_{m} \mu_{1}}\right) \tau^{2}+e^{t_{m} \mu_{1}} \mu_{1}\left(-\left(-1+e^{\tau t_{m}}\right) \mu_{1}+\tau t_{m}\left(\tau+\mu_{1}\right)\right)\right\}\right]+\frac{1}{\mu_{1}^{2}} \mu_{1} D_{\mathrm{MC}} \\
& \times\left[e^{-t_{m}\left(\tau+\mu_{1}\right)}\left\{\frac{e^{t_{2 m} \mu_{1}}\left(\alpha_{1} \mu_{1}+\alpha_{2}\left(-1+t_{2 m} \mu_{1}\right)\right)}{\tau+\mu_{1}}-\frac{e^{t_{m} \mu_{1}}\left(\tau \alpha_{1} \mu_{1}+\alpha_{2}\left(-\tau+\left(1+\tau t_{m}\right) \mu_{1}\right)\right)}{\tau^{2}}\right\}\right. \\
& \left.\left.+e^{-t_{2 m}\left(\tau+\mu_{1}\right)}\left\{-\frac{e^{t_{2 m} \mu_{1}}\left(\alpha_{1} \mu_{1}+\alpha_{2}\left(-1+t_{2 m} \mu_{1}\right)\right)}{\tau+\mu_{1}}+\frac{e^{t_{2 m} \mu_{1}}\left(\tau \alpha_{1} \mu_{1}+\alpha_{2}\left(-\tau+\left(1+\tau t_{2 m}\right) \mu_{1}\right)\right)}{\tau^{2}}\right\}\right]\right] \\
& +\frac{\mathrm{NZ}_{\mathrm{r}}}{\mathrm{T}}\left[O_{\mathrm{rC}}+\frac{P_{\mathrm{rC}}\left(C_{D}-\omega \epsilon\right)}{\mu_{2}}\left[-e^{\mu_{2} t_{1 r}}+e^{\mu_{2} t_{2 r}}\right]+\frac{H_{\mathrm{rrC}}\left(C_{D}-\omega \epsilon\right)}{\mu_{2}}\left(\frac{-1+e^{-\tau t_{r}}}{\tau}+\frac{1-e^{-t_{r}\left(\tau+\mu_{2}\right)}}{\tau+\mu_{2}}\right)+\frac{H_{\mathrm{roC}}}{\tau+\mu_{2}}\right. \\
& \times\left(1-e^{-t_{r}\left(\tau+\mu_{2}\right)}\right) \frac{\left(C_{D}-\omega \epsilon\right)}{\mu_{2}}\left(-e^{\mu_{2} t_{1 r}}+e^{\mu_{2} t_{2 r}}\right)+\frac{H_{\mathrm{roC}}\left(C_{D}-\omega \epsilon\right)}{\mu_{2}}\left(-\frac{e^{-\tau t_{r}}-e^{-\tau t_{2 r}}}{\tau}\right. \\
& \left.+\frac{e^{t_{2 r} \mu_{2}}\left(e^{-t_{r}\left(\tau+\mu_{2}\right)}-e^{-t_{2 r}\left(\tau+\mu_{2}\right)}\right)}{\tau+\mu_{2}}\right)+\mu_{2} D_{\mathrm{rC}}\left[\frac{\left(C_{D}-\omega \epsilon\right)}{\mu_{2}}\left(\frac{-1+e^{-\tau t_{r}}}{\tau}+\frac{1-e^{-t_{r}\left(\tau+\mu_{2}\right)}}{\tau+\mu_{2}}\right)\right. \\
& +\frac{1}{\tau+\mu_{2}}\left(1-e^{-t_{r}\left(\tau+\mu_{2}\right)}\right) \frac{\left(C_{D}-\omega \epsilon\right)}{\mu_{2}}\left(-e^{\mu_{2} t_{1 r}}+e^{\mu_{2} t_{2 r}}\right)+\frac{\left(C_{D}-\omega \epsilon\right)}{\mu_{2}}\left(-\frac{e^{-\tau t_{r}}-e^{-\tau t_{2 r}}}{\tau}\right. \\
& \left.\left.+\frac{e^{t_{2 r} \mu_{2}}\left(e^{-t_{r}\left(\tau+\mu_{2}\right)}-e^{-t_{2 r}\left(\tau+\mu_{2}\right)}\right)}{\tau+\mu_{2}}\right)\right]+\frac{1}{\mathrm{~T}}\left[S_{\mathrm{RC}}+\frac{P_{\mathrm{RC}}}{\tau}\left(1-e^{-\tau t_{R}}\right) P_{R}+H_{\mathrm{RC}} \frac{e^{-t_{R}\left(\tau+\mu_{1}\right)}}{\tau^{2} \mu_{1}^{2}\left(\tau+\mu_{1}\right)}\right. \\
& \times\left[-\tau\left(P_{R}-\alpha_{1}\right) \mu_{1}\left\{\left(-1+e^{t_{R} \mu_{1}}\right) \tau-e^{t_{R} \mu_{1}}\left(-1+e^{\tau t_{R}}\right) \mu_{1}\right\}+\alpha_{2}\left\{-\left(-1+e^{t_{R} \mu_{1}}\right) \tau^{2}+e^{t_{R} \mu_{1}} \mu_{1}\right.\right. \\
& \left.\left.\times\left(-\left(-1+e^{\tau t_{R}}\right) \mu_{1}+\tau t_{R}\left(\tau+\mu_{1}\right)\right)\right\}\right]+\frac{1}{\mu_{1}^{2}} H_{\mathrm{RC}}\left[e ^ { - t _ { R } ( \tau + \mu _ { 1 } ) } \left\{\frac{e^{T \mu_{1}}\left(\alpha_{1} \mu_{1}+\alpha_{2}\left(-1+T \mu_{1}\right)\right)}{\tau+\mu_{1}}\right.\right.
\end{aligned}
$$




$$
\begin{aligned}
& \left.-\frac{e^{t_{R} \mu_{1}}\left(\tau \alpha_{1} \mu_{1}+\alpha_{2}\left(-\tau+\left(1+\tau t_{R}\right) \mu_{1}\right)\right)}{\tau^{2}}\right\}+e^{-T\left(\tau+\mu_{1}\right)}\left\{-\frac{e^{T \mu_{1}}\left(\alpha_{1} \mu_{1}+\alpha_{2}\left(-1+T \mu_{1}\right)\right)}{\tau+\mu_{1}}\right. \\
& \left.\left.+\frac{e^{T \mu_{1}}\left(\tau \alpha_{1} \mu_{1}+\alpha_{2}\left(-\tau+(1+\tau T) \mu_{1}\right)\right)}{\tau^{2}}\right\}\right]+\mu_{1} D_{\mathrm{RC}} \frac{e^{-t_{R}\left(\tau+\mu_{1}\right)}}{\tau^{2} \mu_{1}^{2}\left(\tau+\mu_{1}\right)}\left[-\tau\left(P_{R}-\alpha_{1}\right) \mu_{1}\left\{\left(-1+e^{t_{R} \mu_{1}}\right) \tau\right.\right. \\
& \left.\left.-e^{t_{R} \mu_{1}}\left(-1+e^{\tau t_{R}}\right) \mu_{1}\right\}+\alpha_{2}\left\{-\left(-1+e^{t_{R} \mu_{1}}\right) \tau^{2}+e^{t_{R} \mu_{1}} \mu_{1}\left(-\left(-1+e^{\tau t_{R}}\right) \mu_{1}+\tau t_{R}\left(\tau+\mu_{1}\right)\right)\right\}\right] \\
& +\frac{1}{\mu_{1}^{2}} \mu_{1} D_{\mathrm{RC}}\left[e^{-t_{R}\left(\tau+\mu_{1}\right)}\left\{\frac{e^{T \mu_{1}}\left(\alpha_{1} \mu_{1}+\alpha_{2}\left(-1+T \mu_{1}\right)\right)}{\tau+\mu_{1}}-\frac{e^{t_{R} \mu_{1}}\left(\tau \alpha_{1} \mu_{1}+\alpha_{2}\left(-\tau+\left(1+\tau t_{R}\right) \mu_{1}\right)\right)}{\tau^{2}}\right\}\right. \\
& \left.+e^{-T\left(\tau+\mu_{1}\right)}\left\{-\frac{e^{T \mu_{1}}\left(\alpha_{1} \mu_{1}+\alpha_{2}\left(-1+T \mu_{1}\right)\right)}{\tau+\mu_{1}}+\frac{e^{T \mu_{1}}\left(\tau \alpha_{1} \mu_{1}+\alpha_{2}\left(-\tau+(1+\tau T) \mu_{1}\right)\right)}{\tau^{2}}\right\}\right] \\
& \left.+\frac{C_{\mathrm{RC}}}{\tau}\left(1-e^{-T \tau}\right) \rho \sigma C_{D}\right] \\
& \widetilde{\mathrm{TS}_{C 3}}=\frac{\mathrm{N}}{\mathrm{T}}\left[S_{\mathrm{MC}}+\left(P_{\mathrm{MC}}+\varphi_{4}\right)\left(1-e^{-\tau t_{m}}\right) \frac{P_{M}}{\tau}+H_{\mathrm{MC}} \frac{e^{-t_{M}\left(\tau+\mu_{1}\right)}}{\tau^{2} \mu_{1}^{2}\left(\tau+\mu_{1}\right)}\left[-\tau\left(P_{M}-\alpha_{1}\right) \mu_{1}\left\{\left(-1+e^{t_{m} \mu_{1}}\right) \tau\right.\right.\right. \\
& \left.\left.-e^{t_{m} \mu_{1}}\left(-1+e^{\tau t_{m}}\right) \mu_{1}\right\}+\alpha_{2}\left\{-\left(-1+e^{t_{m} \mu_{1}}\right) \tau^{2}+e^{t_{m} \mu_{1}} \mu_{1}\left(-\left(-1+e^{\tau t_{m}}\right) \mu_{1}+\tau t_{m}\left(\tau+\mu_{1}\right)\right)\right\}\right] \\
& +\frac{1}{\mu_{1}^{2}} H_{\mathrm{MC}}\left[e^{-t_{m}\left(\tau+\mu_{1}\right)}\left\{\frac{e^{t_{2 m} \mu_{1}}\left(\alpha_{1} \mu_{1}+\alpha_{2}\left(-1+t_{2 m} \mu_{1}\right)\right)}{\tau+\mu_{1}}-\frac{e^{t_{m} \mu_{1}}\left(\tau \alpha_{1} \mu_{1}+\alpha_{2}\left(-\tau+\left(1+\tau t_{m}\right) \mu_{1}\right)\right)}{\tau^{2}}\right\}\right. \\
& \left.+e^{-t_{2 m}\left(\tau+\mu_{1}\right)}\left\{-\frac{e^{t_{2 m} \mu_{1}}\left(\alpha_{1} \mu_{1}+\alpha_{2}\left(-1+t_{2 m} \mu_{1}\right)\right)}{\tau+\mu_{1}}+\frac{e^{t_{2 m} \mu_{1}}\left(\tau \alpha_{1} \mu_{1}+\alpha_{2}\left(-\tau+\left(1+\tau t_{2 m}\right) \mu_{1}\right)\right)}{\tau^{2}}\right\}\right] \\
& +\mu_{1} D_{\mathrm{MC}} \frac{e^{-t_{M}\left(\tau+\mu_{1}\right)}}{\tau^{2} \mu_{1}^{2}\left(\tau+\mu_{1}\right)}\left[-\tau\left(P_{M}-\alpha_{1}\right) \mu_{1}\left\{\left(-1+e^{t_{m} \mu_{1}}\right) \tau-e^{t_{m} \mu_{1}}\left(-1+e^{\tau t_{m}}\right) \mu_{1}\right\}\right. \\
& \left.+\alpha_{2}\left\{-\left(-1+e^{t_{m} \mu_{1}}\right) \tau^{2}+e^{t_{m} \mu_{1}} \mu_{1}\left(-\left(-1+e^{\tau t_{m}}\right) \mu_{1}+\tau t_{m}\left(\tau+\mu_{1}\right)\right)\right\}\right]+\frac{1}{\mu_{1}^{2}} \mu_{1} D_{\mathrm{MC}} \\
& \times\left[e^{-t_{m}\left(\tau+\mu_{1}\right)}\left\{\frac{e^{t_{2 m} \mu_{1}}\left(\alpha_{1} \mu_{1}+\alpha_{2}\left(-1+t_{2 m} \mu_{1}\right)\right)}{\tau+\mu_{1}}-\frac{e^{t_{m} \mu_{1}}\left(\tau \alpha_{1} \mu_{1}+\alpha_{2}\left(-\tau+\left(1+\tau t_{m}\right) \mu_{1}\right)\right)}{\tau^{2}}\right\}\right. \\
& \left.\left.+e^{-t_{2 m}\left(\tau+\mu_{1}\right)}\left\{-\frac{e^{t_{2 m} \mu_{1}}\left(\alpha_{1} \mu_{1}+\alpha_{2}\left(-1+t_{2 m} \mu_{1}\right)\right)}{\tau+\mu_{1}}+\frac{e^{t_{2 m} \mu_{1}}\left(\tau \alpha_{1} \mu_{1}+\alpha_{2}\left(-\tau+\left(1+\tau t_{2 m}\right) \mu_{1}\right)\right)}{\tau^{2}}\right\}\right]\right] \\
& +\frac{\mathrm{NZ}_{\mathrm{r}}}{\mathrm{T}}\left[\left(O_{\mathrm{rC}}+\varphi_{2}\right)+\frac{P_{\mathrm{rC}}\left(C_{D}-\left(\omega+\varphi_{8}\right) \epsilon\right)}{\mu_{2}}\left[-e^{\mu_{2} t_{1 r}}+e^{\mu_{2} t_{2 r}}\right]+\frac{H_{\mathrm{rrC}}\left(C_{D}-\left(\omega+\varphi_{8}\right) \epsilon\right)}{\mu_{2}}\left(\frac{-1+e^{-\tau t_{r}}}{\tau}\right.\right. \\
& \left.+\frac{1-e^{-t_{r}\left(\tau+\mu_{2}\right)}}{\tau+\mu_{2}}\right)+\frac{H_{\mathrm{roC}}}{\tau+\mu_{2}}\left(1-e^{-t_{r}\left(\tau+\mu_{2}\right)}\right) \frac{\left(C_{D}-\left(\omega+\varphi_{8}\right) \epsilon\right)}{\mu_{2}}\left(-e^{\mu_{2} t_{1 r}}+e^{\mu_{2} t_{2 r}}\right)+\frac{H_{\mathrm{roC}}\left(C_{D}-\left(\omega+\varphi_{8}\right) \epsilon\right)}{\mu_{2}} \\
& \times\left(-\frac{e^{-\tau t_{r}}-e^{-\tau t_{2 r}}}{\tau}+\frac{e^{t_{2 r} \mu_{2}}\left(e^{-t_{r}\left(\tau+\mu_{2}\right)}-e^{-t_{2 r}\left(\tau+\mu_{2}\right)}\right)}{\tau+\mu_{2}}\right)+\mu_{2} D_{\mathrm{rC}}\left[\frac { ( C _ { D } - ( \omega + \varphi _ { 8 } ) \epsilon ) } { \mu _ { 2 } } \left(\frac{-1+e^{-\tau t_{r}}}{\tau}\right.\right. \\
& \left.+\frac{1-e^{-t_{r}\left(\tau+\mu_{2}\right)}}{\tau+\mu_{2}}\right)+\frac{1}{\tau+\mu_{2}}\left(1-e^{-t_{r}\left(\tau+\mu_{2}\right)}\right) \frac{\left(C_{D}-\left(\omega+\varphi_{8}\right) \epsilon\right)}{\mu_{2}}\left(-e^{\mu_{2} t_{1 r}}+e^{\mu_{2} t_{2 r}}\right)+\frac{\left(C_{D}-\left(\omega+\varphi_{8}\right) \epsilon\right)}{\mu_{2}} \\
& \left.\times\left(-\frac{e^{-\tau t_{r}}-e^{-\tau t_{2 r}}}{\tau}+\frac{e^{t_{2 r} \mu_{2}}\left(e^{-t_{r}\left(\tau+\mu_{2}\right)}-e^{-t_{2 r}\left(\tau+\mu_{2}\right)}\right)}{\tau+\mu_{2}}\right)\right]+\frac{1}{\mathrm{~T}}\left[S_{\mathrm{RC}}+\frac{\left(P_{\mathrm{RC}}+\varphi_{6}\right)}{\tau}\left(1-e^{-\tau t_{R}}\right) P_{R}\right. \\
& +H_{\mathrm{RC}} \frac{e^{-t_{R}\left(\tau+\mu_{1}\right)}}{\tau^{2} \mu_{1}^{2}\left(\tau+\mu_{1}\right)}\left[-\tau\left(P_{R}-\alpha_{1}\right) \mu_{1}\left\{\left(-1+e^{t_{R} \mu_{1}}\right) \tau-e^{t_{R} \mu_{1}}\left(-1+e^{\tau t_{R}}\right) \mu_{1}\right\}+\alpha_{2}\left\{-\left(-1+e^{t_{R} \mu_{1}}\right) \tau^{2}\right.\right. \\
& \left.\left.+e^{t_{R} \mu_{1}} \mu_{1}\left(-\left(-1+e^{\tau t_{R}}\right) \mu_{1}+\tau t_{R}\left(\tau+\mu_{1}\right)\right)\right\}\right]+\frac{1}{\mu_{1}^{2}} H_{\mathrm{RC}}\left[e ^ { - t _ { R } ( \tau + \mu _ { 1 } ) } \left\{\frac{e^{T \mu_{1}}\left(\alpha_{1} \mu_{1}+\alpha_{2}\left(-1+T \mu_{1}\right)\right)}{\tau+\mu_{1}}\right.\right.
\end{aligned}
$$




$$
\begin{aligned}
& \left.-\frac{e^{t_{R} \mu_{1}}\left(\tau \alpha_{1} \mu_{1}+\alpha_{2}\left(-\tau+\left(1+\tau t_{R}\right) \mu_{1}\right)\right)}{\tau^{2}}\right\}+e^{-T\left(\tau+\mu_{1}\right)}\left\{-\frac{e^{T \mu_{1}}\left(\alpha_{1} \mu_{1}+\alpha_{2}\left(-1+T \mu_{1}\right)\right)}{\tau+\mu_{1}}\right. \\
& \left.\left.+\frac{e^{T \mu_{1}}\left(\tau \alpha_{1} \mu_{1}+\alpha_{2}\left(-\tau+(1+\tau T) \mu_{1}\right)\right)}{\tau^{2}}\right\}\right]+\mu_{1} D_{\mathrm{RC}} \frac{e^{-t_{R}\left(\tau+\mu_{1}\right)}}{\tau^{2} \mu_{1}^{2}\left(\tau+\mu_{1}\right)}\left[-\tau\left(P_{R}-\alpha_{1}\right) \mu_{1}\left\{\left(-1+e^{t_{R} \mu_{1}}\right) \tau\right.\right. \\
& \left.\left.-e^{t_{R} \mu_{1}}\left(-1+e^{\tau t_{R}}\right) \mu_{1}\right\}+\alpha_{2}\left\{-\left(-1+e^{t_{R} \mu_{1}}\right) \tau^{2}+e^{t_{R} \mu_{1}} \mu_{1}\left(-\left(-1+e^{\tau t_{R}}\right) \mu_{1}+\tau t_{R}\left(\tau+\mu_{1}\right)\right)\right\}\right] \\
& +\frac{1}{\mu_{1}^{2}} \mu_{1} D_{\mathrm{RC}}\left[e^{-t_{R}\left(\tau+\mu_{1}\right)}\left\{\frac{e^{T \mu_{1}}\left(\alpha_{1} \mu_{1}+\alpha_{2}\left(-1+T \mu_{1}\right)\right)}{\tau+\mu_{1}}-\frac{e^{t_{R} \mu_{1}}\left(\tau \alpha_{1} \mu_{1}+\alpha_{2}\left(-\tau+\left(1+\tau t_{R}\right) \mu_{1}\right)\right)}{\tau^{2}}\right\}\right. \\
& \left.+e^{-T\left(\tau+\mu_{1}\right)}\left\{-\frac{e^{T \mu_{1}}\left(\alpha_{1} \mu_{1}+\alpha_{2}\left(-1+T \mu_{1}\right)\right)}{\tau+\mu_{1}}+\frac{e^{T \mu_{1}}\left(\tau \alpha_{1} \mu_{1}+\alpha_{2}\left(-\tau+(1+\tau T) \mu_{1}\right)\right)}{\tau^{2}}\right\}\right] \\
& \left.+\frac{C_{\mathrm{RC}}}{\tau}\left(1-e^{-T \tau}\right) \rho \sigma C_{D}\right] .
\end{aligned}
$$

\section{Optimal solution procedure}

This study mainly focuses on minimizing the total cost functions $\mathrm{TS}_{C}$ and $\widetilde{\mathrm{TS}_{C}^{\prime}}$ which are given in equations (4.17) and (4.18).

For a crisp model, from equation (4.17), we obtain

$$
\frac{\partial \mathrm{TS}_{C}}{\partial t_{m}}=0 \quad \& \quad \frac{\partial \mathrm{TS}_{C}}{\partial t_{R}}=0 .
$$

Determining the optimal values of $t_{m}^{*}$ and $t_{R}^{*}$ is not easy; thus, the Mathematica software is used to determine the optimal values.

The Hessian matrix,

$$
H_{C}=\operatorname{det}\left(\begin{array}{cc}
\frac{\partial^{2} \mathrm{TS}_{C}}{\partial t_{m}^{2}} & \frac{\partial^{2} \mathrm{TS}_{C}}{\partial t_{m} \partial t_{R}} \\
\frac{\partial^{2} \mathrm{TS}_{C}}{\partial t_{m} \partial t_{R}} & \frac{\partial^{2} \mathrm{TS}_{C}}{\partial t_{R}^{2}}
\end{array}\right)>0 \quad \text { and } \quad \frac{\partial^{2} \mathrm{TS}_{C}}{\partial t_{m}^{2}}>0, \frac{\partial^{2} \mathrm{TS}_{C}}{\partial t_{R}^{2}}>0
$$

satisfies the sufficient condition of the minimizing function.

Then, this study obtains the value of the total minimum-cost function from equation (4.17).

The fuzzy model is processed in a similar manner.

\section{Algorithm}

(1) First, this study considers the value of the total cost function $t_{2 M}=\frac{T}{N}, t_{2 r}=\frac{T}{N Z_{r}}$, and $t_{2 r}=2 t_{r}$. And determine the total cost function as $\mathrm{TS}_{C}\left(t_{m}, t_{R}\right)$.

(2) Then, this study considers the necessary conditions $\frac{\partial \mathrm{TS}_{C}}{\partial t_{m}}=0$ and $\frac{\partial \mathrm{TS}_{C}}{\partial t_{R}}=0$, and determine the values of $t_{m}^{*}$ and $t_{R}^{*}$.

(3) The sufficient condition satisfies the Hessian matrix, as follows:

$$
H_{C}=\operatorname{det}\left(\begin{array}{cc}
\frac{\partial^{2} \mathrm{TS}_{C}}{\partial t_{m}^{2}} & \frac{\partial^{2} \mathrm{TS}_{C}}{\partial t_{m} \partial t_{R}} \\
\frac{\partial^{2} \mathrm{TS}_{C}}{\partial t_{m} \partial t_{R}} & \frac{\partial^{2} \mathrm{TS}_{C}}{\partial t_{R}^{2}}
\end{array}\right)>0 \quad \text { and } \quad \frac{\partial^{2} \mathrm{TS}_{C}}{\partial t_{m}^{2}}>0, \quad \frac{\partial^{2} \mathrm{TS}_{C}}{\partial t_{R}^{2}}>0 .
$$

(4) Then, this study obtains the values of $t_{2 M}, t_{r}$, and $t_{2 r}$.

(5) Finally, this study obtains the minimization value of the total cost function. 
TABLE 2. Optimal result of crisp and fuzzy environments.

\begin{tabular}{llll}
\hline \hline & Total cost & $t_{m}$ & $t_{R}$ \\
\hline Crisp environment & 7055.17 & 0.2249 & 0.9609 \\
Fuzzy environment & 7031.29 & 0.2252 & 0.9609 \\
\hline
\end{tabular}

\section{Numerical EXAMPLE}

In this section, the proposed model is demonstrated through an illustrative numerical example.

\section{Crisp environment}

Example 5.1. This study considers the parameters $\alpha_{1}=11, \alpha_{2}=1, \mu_{1}=21, N=3, S_{\mathrm{LMC}}=45, P_{\mathrm{MC}}=42$, $H_{\mathrm{MC}}=21, D_{\mathrm{LMC}}=41, P_{M}=56, \tau=0.5, O_{\mathrm{rC}}=35, P_{\mathrm{LrC}}=31, H_{\mathrm{rrC}}=51, H_{\mathrm{roC}}=25, D_{\mathrm{LrC}}=26, C_{D}=71$, $Z_{r}=8, \mu_{2}=25, S_{\mathrm{LRC}}=35, P_{\mathrm{RC}}=45, P_{R}=15, H_{\mathrm{RC}}=30, D_{\mathrm{LRC}}=58, C_{\mathrm{RC}}=31, \rho=0.3, \sigma=0.1$, $T=1, S_{1}=1, S_{2}=1, P_{1}=1, D_{1}=1, D_{2}=2, D_{3}=3, \Delta=3, K=0.2, \omega=0.2, \epsilon=0.3$. This study uses the Mathematica software to satisfy the minimization condition $\operatorname{det}\left(\begin{array}{cc}266051 & 21783.4 \\ 21783.4 & 37.3628\end{array}\right)>5.795 \times 10^{9}>0$ and $\frac{\partial^{2} \mathrm{TS}_{C}}{\partial t_{m}^{2}}>266051>0, \frac{\partial^{2} \mathrm{TS}_{C}}{\partial t_{R}^{2}}>37.3628>0$, for the optimal result and obtain $t_{m}=0.2249, t_{2 m}=0.333$, $t_{r}=0.0208, t_{2 r}=0.0416, t_{R}=0.9609$, with a total cost of 7055.17 .

\section{Fuzzy environment}

Example 5.2. This study considers the parameters $\varphi_{1}=4, \varphi_{2}=3, \varphi_{3}=5, \varphi_{4}=3, \varphi_{5}=6$, $\varphi_{6}=6, \varphi_{7}=0.02, \varphi_{8} \rightarrow 0.02$, and apply the Mathematica software to satisfy the minimization condition. $\operatorname{det}\left(\begin{array}{cc}266088 & 21783.4 \\ 21783.4 & 37.3628\end{array}\right)>5.795 \times 10^{9}>0$ and $\frac{\partial^{2} \mathrm{TS}_{C}}{\partial t_{m}^{2}}>266088>0, \frac{\partial^{2} \mathrm{TS}_{C}}{\partial t_{R}^{2}}>37.3628>0$. This study obtains the following result $t_{m}=0.2252, t_{2 m}=0.333, t_{r}=0.0208, t_{2 r}=0.0416, t_{R}=0.9609$, and a total cost of 7031.29 .

The optimal result of crisp and fuzzy environment represented in Table 2.

\section{Sensitivity Analysis}

In this section, this study discusses the percentage change in the demand rate, holding cost, the production cost of manufacturing and remanufacturing, and demand rate and holding cost of the retailer, owing to which an effective change was observed in the total cost.

\section{Observations and managerial insights}

The crisp environment results are presented in Tables 3 and 5, and Figures 4, 5, and 8, whereas the fuzzy environment results are presented in Tables 4 and 6 and Figures 6, 7, and 9. These study's observations and managerial insights are as follows:

(i) From Figures 4 and 6, a reduction in the total cost was observed when there was a percentage change $(-20,-10,0,+10$, and $+20 \%)$ in the first demand parameter $\alpha_{1}$. The percentage change $(-20,-10$, $0,+10$, and $+20 \%)$ in the second demand parameter $\alpha_{2}$ increased the total cost. The manager should take care about the learning effect because using learning, the total cost can be reduced more than the traditional model without learning.

(ii) From Figures 4 and 6 , it can be observed that the total cost increases after a percentage change $(-20$, $-10,0,+10$, and $+20 \%$ ) in the holding cost parameters $H_{\mathrm{MC}}$ and $H_{\mathrm{RC}}$. 
TABLE 3. Change in total cost due to percentage change in parameters in crisp environment.

\begin{tabular}{llllll}
\hline \hline & \multicolumn{5}{c}{ Total cost } \\
\cline { 2 - 6 } Parameters & $-20 \%$ & $-10 \%$ & $0 \%$ & $+10 \%$ & $+20 \%$ \\
\hline$\alpha_{1}$ & 7107.28 & 7084.99 & 7055.17 & 7019.23 & 6978.21 \\
$\alpha_{2}$ & 7046.02 & 7050.60 & 7055.17 & 7059.73 & 7064.29 \\
$H_{\mathrm{MC}}$ & 7048.84 & 7052.00 & 7055.17 & 7058.33 & 7061.50 \\
$H_{\mathrm{rrC}}$ & 7058.34 & 7056.75 & 7055.17 & 7053.58 & 7052.00 \\
$H_{\mathrm{roC}}$ & 7144.20 & 7099.68 & 7055.17 & 7010.65 & 6966.14 \\
$H_{\mathrm{RC}}$ & 7054.39 & 7054.78 & 7055.17 & 7055.56 & 7055.95 \\
$\epsilon$ & 7055.57 & 7055.37 & 7055.17 & 7054.97 & 7054.76 \\
$C_{D}$ & 6565.14 & 6810.16 & 7055.17 & 7300.18 & 7545.19 \\
$P_{M}$ & 6502.31 & 6782.1 & 7055.17 & 7322.18 & 7583.68 \\
$P_{R}$ & 6818.28 & 6936.97 & 7055.17 & 7172.91 & 7290.24 \\
\hline
\end{tabular}

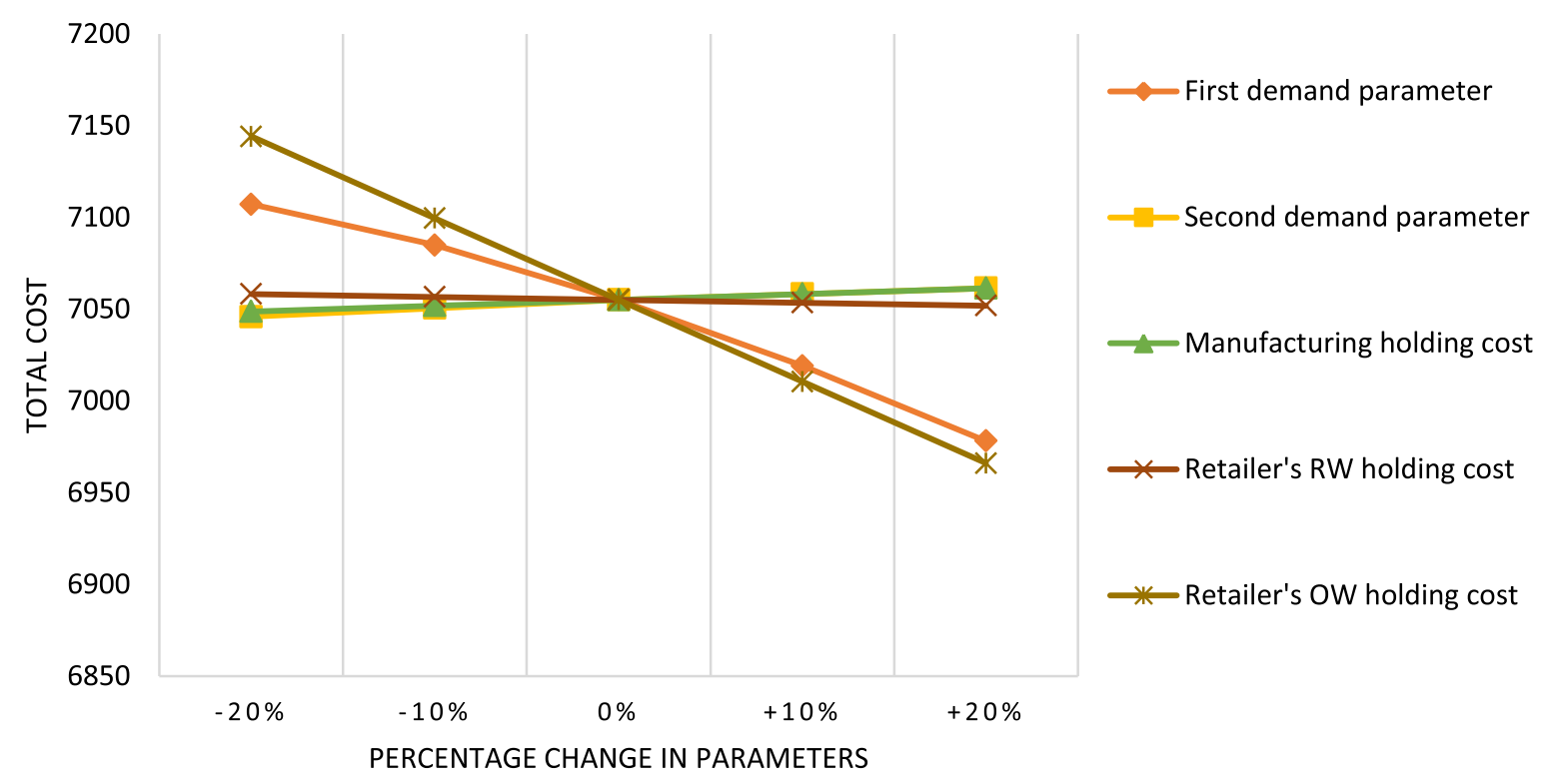

Figure 4 . Change in total cost due to the percentage change in $\alpha_{1}, \alpha_{2}, H_{\mathrm{MC}}, H_{\mathrm{rrC}}$ and $H_{\mathrm{roC}}$ parameters in crisp environment.

(iii) From Figures 5 and 7 , the total cost volume was observed to increase due to the percentage change $(-20$, $-10,0,+10$, and $+20 \%$ ) in the production parameters $P_{M}$ and $P_{R}$.

(iv) From Figures 4 and 6 , a decrease in the total cost was observed when the percentage change $(-20,-10$, $0,+10$, and $+20 \%)$ in the holding cost parameters $H_{\mathrm{rrC}}$ and $H_{\mathrm{roC}}$ of RW and $\mathrm{OW}$ changed from negative to positive.

(v) From Figures 5 and 7, an increase in the total cost was observed when there was a percentage change $(-20,-10,0,+10$, and $+20 \%)$ in the demand parameter $C_{D}$.

(vi) From Figures 5 and 7 , the total cost was observed to decrease with the percentage change $(-20,-10,0$, +10 , and $+20 \%$ ) in the carbon emissions parameter $\epsilon$.

(vii) The total cost decreases for each new shipment and learning rate, as depicted in Figures 8 and 9. 


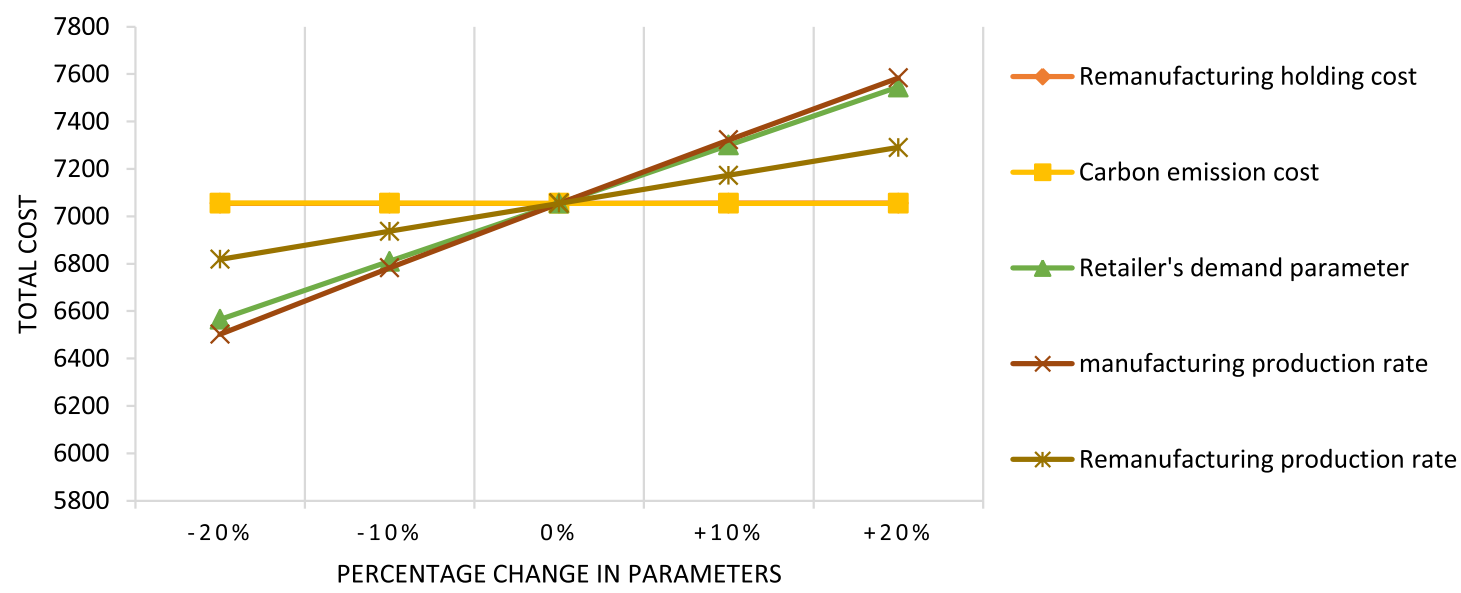

Figure 5. Change in total cost due to percentage change in $H_{\mathrm{RC}}, \epsilon, C_{D}, P_{M}$ and $P_{R}$ parameters in crisp environment.

TABLE 4. Change in total cost due to percentage change in parameters in fuzzy environment.

\begin{tabular}{llllll}
\hline \hline & \multicolumn{5}{c}{ Total cost } \\
\cline { 2 - 6 } Parameters & $-20 \%$ & $-10 \%$ & $0 \%$ & $+10 \%$ & $+20 \%$ \\
\hline$\alpha_{1}$ & 7084.17 & 7061.47 & 7031.29 & 6995.021 & 6953.69 \\
$\alpha_{2}$ & 7022.16 & 7026.73 & 7031.29 & 7035.84 & 7040.38 \\
$H_{\mathrm{MC}}$ & 7024.96 & 7028.13 & 7031.29 & 7034.45 & 7037.6 \\
$H_{\mathrm{rrC}}$ & 7034.46 & 7032.87 & 7031.29 & 7029.7 & 7028.12 \\
$H_{\mathrm{roC}}$ & 7120.31 & 7075.8 & 7031.29 & 6986.77 & 6942.26 \\
$H_{\mathrm{RC}}$ & 7030.91 & 7030.9 & 7031.29 & 7031.67 & 7032.06 \\
$\epsilon$ & 7031.69 & 7031.49 & 7031.29 & 7031.08 & 7030.88 \\
$C_{D}$ & 6541.26 & 6782.82 & 7031.29 & 7276.30 & 7521.31 \\
$P_{M}$ & 6681.37 & 6759.67 & 7031.29 & 7296.88 & 7575.39 \\
$P_{R}$ & 6794.40 & 6913.09 & 7031.29 & 7149.03 & 7266.36 \\
\hline
\end{tabular}

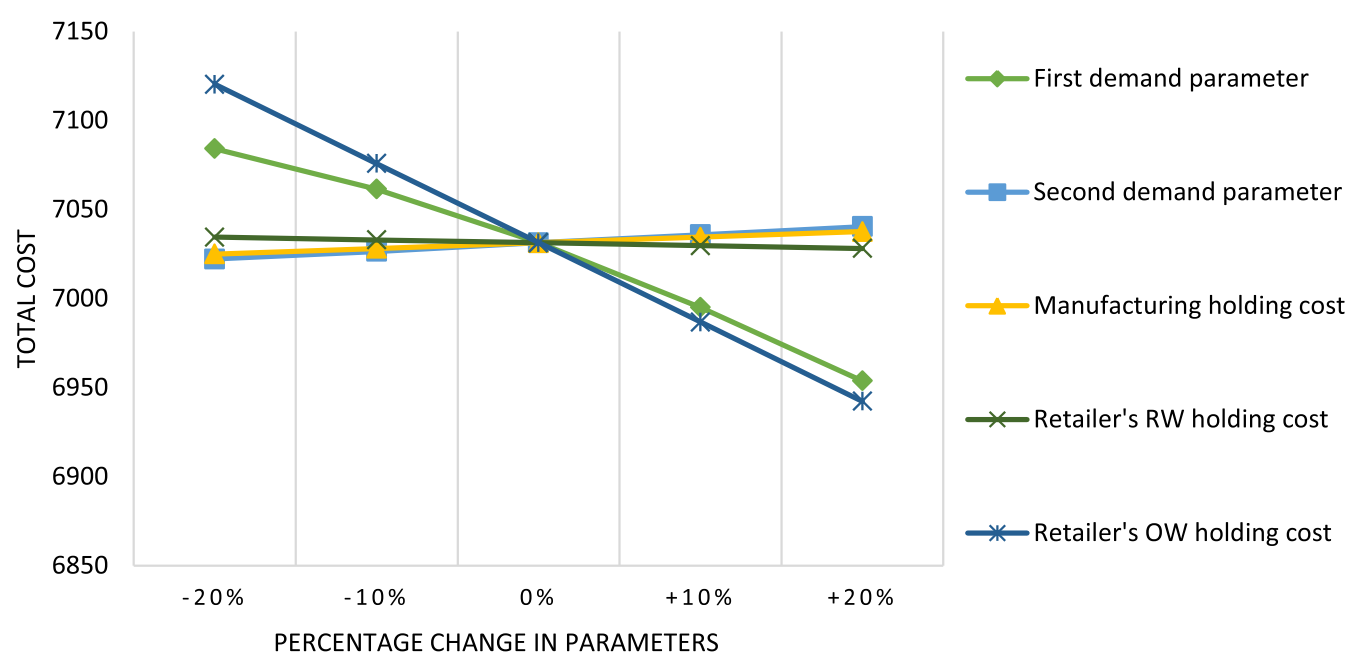

Figure 6 . Change in total cost due to the percentage change in $\alpha_{1}, \alpha_{2}, H_{\mathrm{MC}}, H_{\mathrm{rrC}}$ and $H_{\mathrm{roC}}$ parameters in fuzzy environment. 


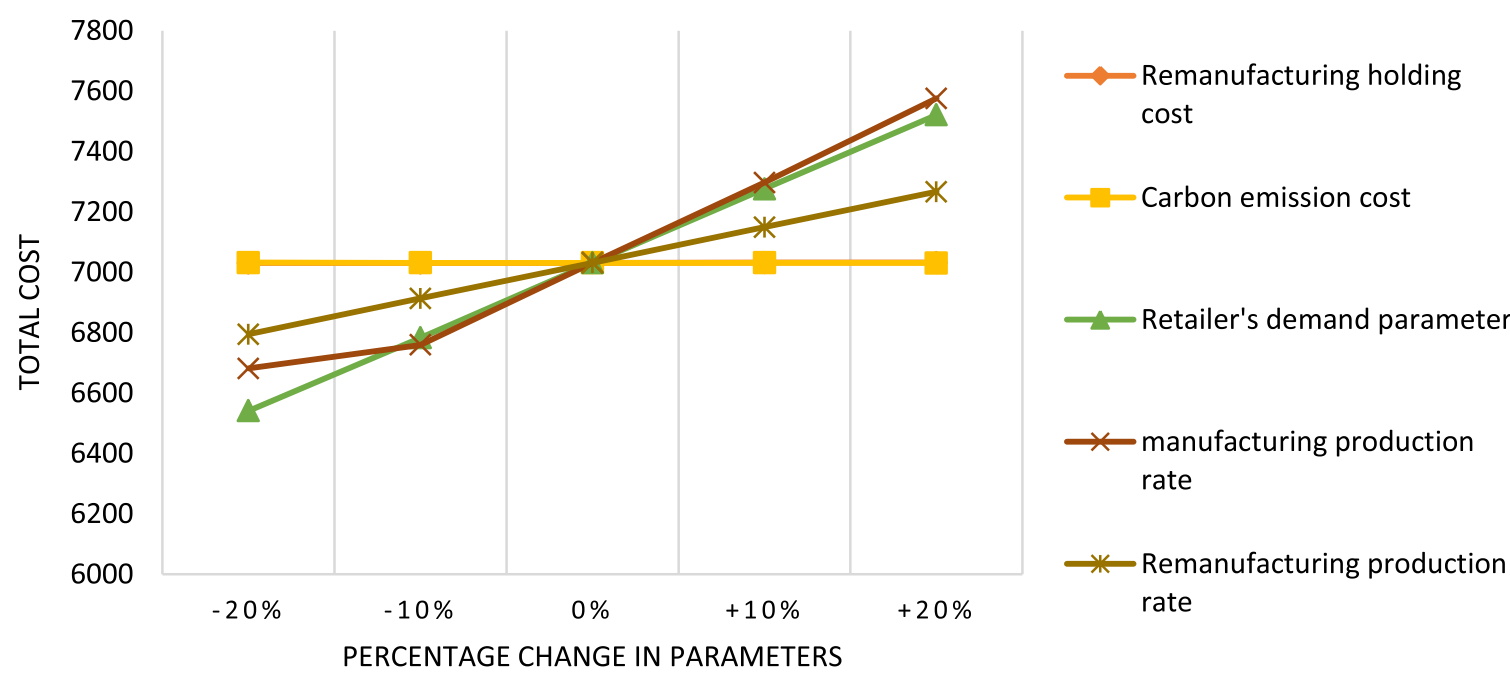

Figure 7 . Change in total cost due to the percentage change in $H_{\mathrm{RC}}, \epsilon, C_{D}, P_{M}$ and $P_{R}$ parameters in fuzzy environment.

TABle 5. Total cost reduction due to learning effect in crisp environment.

\begin{tabular}{lllll}
\hline \hline & \multicolumn{4}{c}{ Effective total cost } \\
\cline { 2 - 5 } No. of shipments $\Delta$ & 0.2 & 0.4 & 0.6 & 0.8 \\
\hline 2 & 7066.66 & 7047.56 & 7030.94 & 7016.47 \\
3 & 7055.17 & 7026.34 & 7006.8 & 6989.5 \\
4 & 7047.56 & 7016.47 & 6992.9 & 6975.04 \\
5 & 7041.96 & 7008.16 & 6983.65 & 6965.9 \\
\hline
\end{tabular}

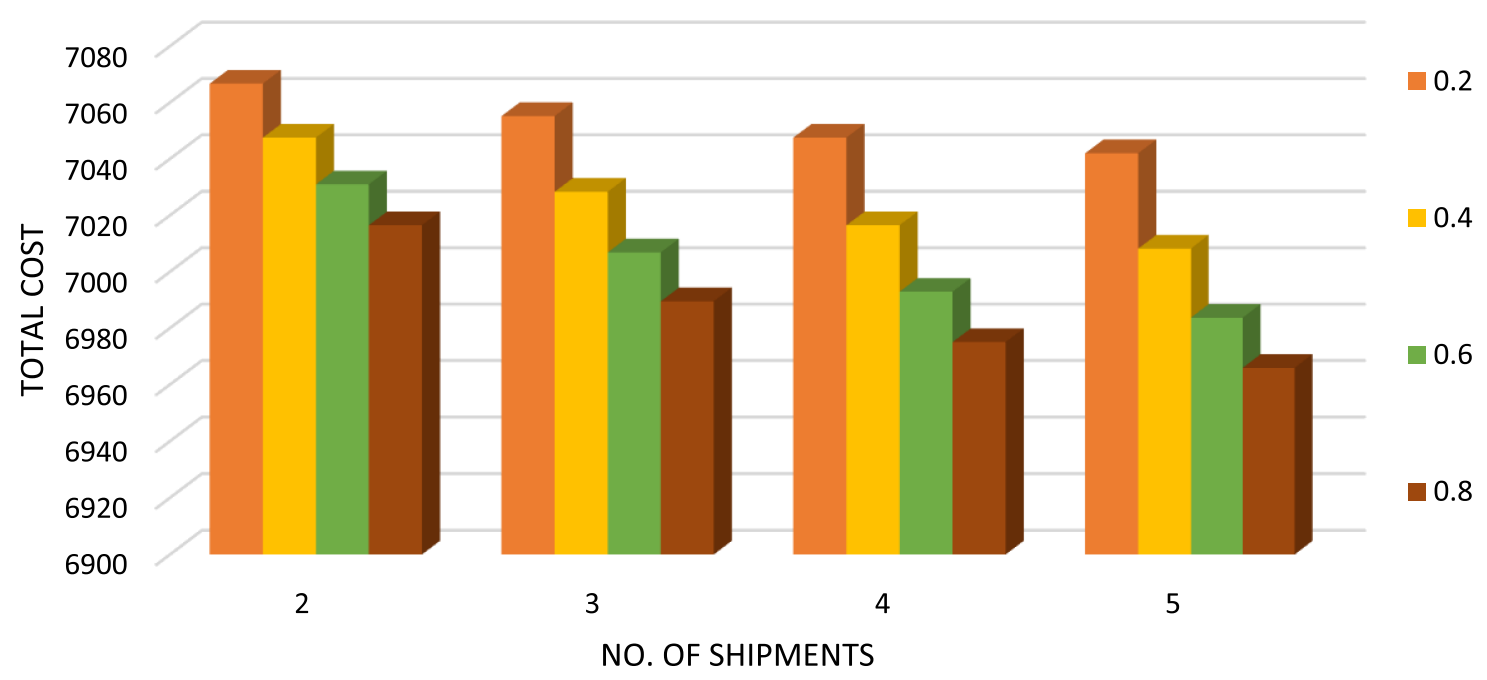

Figure 8. Change in total cost due to learning effect in crisp environment. 
TABLE 6 . Total cost reduction due to learning effect in fuzzy environment.

\begin{tabular}{lllll}
\hline \hline & \multicolumn{4}{c}{ Effective total cost } \\
\cline { 2 - 5 } No. of shipments $\Delta$ & 0.2 & 0.4 & 0.6 & 0.8 \\
\hline 2 & 7042.77 & 7023.68 & 7007.06 & 6992.59 \\
3 & 7031.29 & 7004.46 & 6982.93 & 6965.64 \\
4 & 7023.68 & 6992.59 & 6969.03 & 6951.17 \\
5 & 7018.08 & 6984.29 & 6959.79 & 6942.03 \\
\hline
\end{tabular}

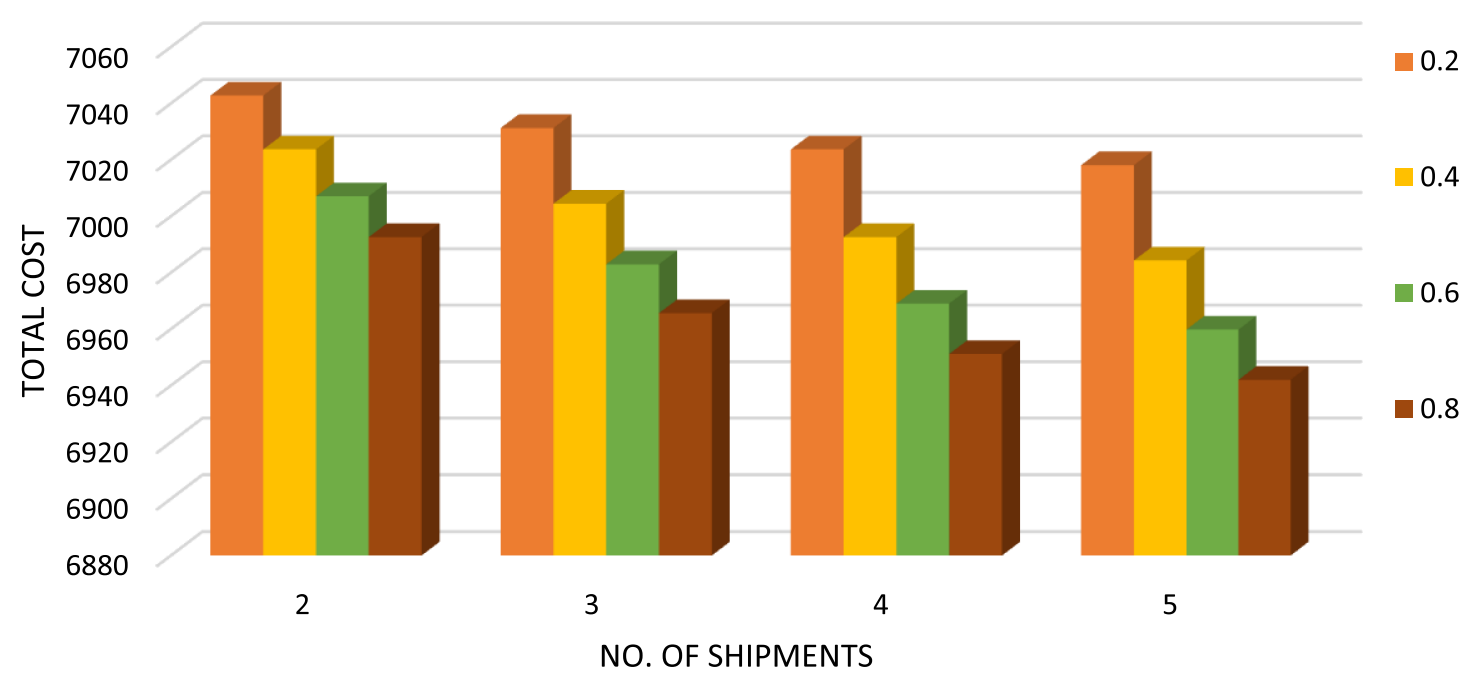

Figure 9. Change in total cost due to learning effect in fuzzy environment.

\section{Conclusions}

In this model, two retailing warehouses were considered. Customers are considered to have a demand for smart products. In the event of receiving defective products, the customers return the products, which then undergo the remanufacturing process. This reverse supply chain model under inflation and the retailer's two warehouses are critical for the market. Moreover, the result of the reverse logistics model is significant in today's market. The learning effect influences the setup cost (i.e., the costs of labor, machines, change of tool, etc.) and deterioration rate. A decrease in the total cost is observed due to the learning effect, as depicted in Figures 8 and 9. The fuzzy theory's SDM is used to manage this uncertainty in the ordering cost, production cost, reproduction cost, and carbon emission demand parameter. The manufacturer establishes a competitive partnership with mutually beneficial aims to cope with smart products and create a carbon emissions market. Sensitivity analysis and graphical illustrations have been widely examined for a more significant validation of the model. There are certain limitations to this proposed study, which essentially constitutes research on several reverse logistics services and strategies in the electronics industry and some other industries, but not for all. In production, remanufacturing, and return planning, this study can be a valuable contribution for decisionmakers. In the future, this study can be developed by considering various other factors such as trade credit, volume flexibility, the effect of a machine breakdown, and investment in green technology to reduce carbon emissions and deterioration. 
Acknowledgements. The work is supported by the National Research Foundation of Korea (NRF) grant, funded by the Korea Government (MSIT) (NRF-2020R1F1A1064460). The first author Subhash Kumar is working at Meerut College,Meerut as a research scholar and thankful to UGC, India, for the financial support.

\section{REFERENCES}

[1] Q. Bai, Y.Y. Gong, M. Jin and X. Xu, Effects of carbon emission reduction on supply chain coordination with vendor-managed deteriorating product inventory. Int. J. Prod. Econ. 208 (2018) 83-99.

[2] R. Batarfi, M.Y. Jaber and C.H. Glock, Pricing and inventory decisions in a dual-channel supply chain with learning and forgetting. Comput. Ind. Eng. 136 (2019) 397-420.

[3] M. Bernon and J. Cullen, An integrated approach to managing reverse logistics. Int. J. Logistics Res. App. 10 (2007) 41-56.

[4] R. Chakrabarty, T. Roy and K.S. Chaudhuri, A two-warehouse inventory model for deteriorating items with capacity constraints and back-ordering under financial considerations. Int. J. Appl. Comput. Math. 4 (2018) 58.

[5] M. Chouinard, S. D'Amours and D. Aït-Kadi, Integration of reverse logistics activities within a supply chain information system. Comput. Ind. 56 (2005) 105-124.

[6] K.-J. Chung, C.-C. Her and S.-D. Lin, A two-warehouse inventory model with imperfect quality production processes. Comput. Ind. Eng. 56 (2009) 193-197.

[7] C.-Y. Dye and C.-T. Yang, Sustainable trade credit and replenishment decisions with credit-linked demand under carbon emission constraints. Eur. J. Oper. Res. 244 (2015) 187-200.

[8] Y. Ghiamia and P. Beullens, The continuous resupply policy for deteriorating items with stock-dependent observable demand in a two-warehouse and two-echelon supply chain. Appl. Math. Modell. 82 (2020) 271-292.

[9] B.C. Giri and C.H. Glock, A closed-loop supply chain with stochastic product returns and worker experience under learning and forgetting. Int. J. Prod. Res. 55 (2017) 6760-6778.

[10] B.C. Giri and M. Masanta, Developing a closed-loop supply chain model with price and quality dependent demand and learning in production in a stochastic environment. Int. J. Syst. Sci.: Oper. Logistics 7 (2020) 147-163.

[11] Y.-S. Huang, C.-C. Fang and Y.-A. Lin, Inventory management in supply chains with consideration of logistics, green investment and different carbon emissions policies. Comput. Ind. Eng. 139 (2019) 106207.

[12] P. Jawla and S.R. Singh, A reverse logistic inventory model for imperfect production process with preservation technology investment under learning and inflationary environment. Uncertain Suppl. Chain Manage. 4 (2016) 107-122.

[13] W.F. Khan and O. Dey, Periodic review inventory model with normally distributed fuzzy random variable demand. Int. J. Syst. Sci.: Oper. Logistics 6 (2017) 1-11.

[14] M.A. Khan, A.A. Shaikh, G.C. Panda and I. Konstantaras, Two-warehouse inventory model for deteriorating items with partial backlogging and advance payment scheme. RAIRO:OR 53 (2019) 1691-1708.

[15] M.A.-A. Khan, A.A. Shaikh, G.C. Panda, A.K. Bhunia and I. Konstantaras, Non-instantaneous deterioration effect in ordering decisions for a two-warehouse inventory system under advance payment and backlogging. Ann. Oper. Res. 289 (2020) $243-275$.

[16] R.S. Kumar, M.K. Tiwari and A. Goswami, Two-echelon fuzzy stochastic supply chain for the manufacturer-buyer integrated production-inventory system. J. Intell. Manuf. 27 (2014) 875-888.

[17] S. Kumar, A. Kumar and M. Jain, Learning effect on an optimal policy for mathematical inventory model for decaying items under preservation technology with the environment of COVID-19 pandemic. Malaya J. Matematik 8 (2020) $1694-1702$.

[18] T.-Y. Liao, Reverse logistics network design for product recovery and remanufacturing. Appl. Math. Model. 60 (2018) 145-163.

[19] J.-J. Liao, K.-J. Chung and K.-N. Huang, A deterministic inventory model for deteriorating items with two warehouses and trade credit in a supply chain system. Int. J. Prod. Econ. 146 (2013) 557-565.

[20] A.K. Manna, J.K. Dey and S.K. Mondal, Three-layer supply chain in an imperfect production inventory model with two storage facilities under fuzzy rough environment. J. Uncertainty Anal. App. 2 (2014) 17.

[21] P. Majumder, U.K. Bera and M. Maiti, An EPQ model for two-warehouse in unremitting release pattern with two-level trade credit period concerning both supplier and retailer. Appl. Math. Comput. 274 (2016) 430-458.

[22] J. Maric and M. Opazo-Basáez, Green servitization for flexible and sustainable supply chain operations: a review of reverse logistics services in manufacturing. Global J. Flexible Syst. Manage. 20 (2019) S65-S80.

[23] U. Mishra, J.-Z. Wu and B. Sarkar, A sustainable production-inventory model for a controllable carbon emissions rate under shortages. J. Cleaner Prod. 256 (2020) 120268.

[24] J. Noh and J.S. Kim, Cooperative green supply chain management with greenhouse gas emissions and fuzzy demand. J. Cleaner Prod. 208 (2019) 1421-1435.

[25] S. Rani, R. Ali and A. Agarwal, Fuzzy inventory model for deteriorating items in a green supply chain with carbon concerned demand. OPSEARCH $\mathbf{5 6}$ (2019) 91-122.

[26] S. Rani, R. Ali and A. Agarwal, Inventory model for deteriorating items in green supply chain with credit period dependent demand. Int. J. Appl. Eng. Res. 15 (2020) 157-172.

[27] S. Rani, R. Ali and A. Agarwal, Fuzzy inventory model for new and refurbished deteriorating items with cannibalisation in green supply chain. Int. J. Syst. Sci.: Oper. Logistics (2020) 1-17. DOI: 10.1080/23302674.2020.1803434.

[28] A. Roy, K. Maity, S. Kar and M. Maiti, A production-inventory model with remanufacturing for defective and usable items in fuzzy-environment. Comput. Ind. Eng. 56 (2009) 87-96. 
[29] B. Sarkar, Mathematical and analytical approach for the management of defective items in a multi-stage production system. J. Cleaner Prod. 218 (2019) 896-919.

[30] B. Sarkar, M. Ullah and N. Kim, Environmental and economic assessment of closed-loop supply chain with remanufacturing and returnable transport items. Comput. Ind. Eng. 111 (2017) 148-163.

[31] B. Sarkar, M. Tayyab, N. Kim and M.S. Habib, Optimal production delivery policies for supplier and manufacturer in a constrained closed-loop supply chain for returnable transport packaging through metaheuristic approach. Comput. Ind. Eng. 135 (2019) 987-1003.

[32] B. Sarkar, M. Sarkar, B. Ganguly and L.E. Cardenas-Barron, Combined effects of carbon emission and production quality improvement for fixed lifetime products in a sustainable supply chain management. Int. J. Prod. Econ. (2020) 107867.

[33] N. Safdar, R. Khalid, W. Ahmed and M. Imran, Reverse logistics network design of e-waste management under the triple bottom line approach. J. Cleaner Prod. 272 (2020) 122662.

[34] C. Singh and S.R. Singh, Supply chain model for expiring items following ramp-type demand with stochastic lead time under crisp and fuzzy environment. Int. J. Fuzzy Syst. App. 9 (2020) 64-91.

[35] S.R. Singh and S. Sharma, A partially backlogged supply chain model for deteriorating items under reverse logistics, imperfect production/remanufacturing and inflation. Int. J. Logistics Syst. Manage. 33 (2019) 221.

[36] S.R. Singh, S. Sharma and M. Kumar, A reverse logistics model for decaying items with variable production and remanufacturing incorporating learning effects. Int. J. Oper. Res. 38 (2020) 422.

[37] N. Saxena, S.R. Singh and S.S. Sana, A green supply chain model of vendor and buyer for remanufacturing. RAIRO:OR 51 (2017) 1133-1150.

[38] N. Saxena, B. Sarkar and S.R. Singh, Selection of remanufacturing/production cycles with an alternative market: a perspective on waste management. J. Cleaner Prod. 245 (2020) 118935.

[39] M. Tayyab, B. Sarkar and B. Yahya, Imperfect multi-stage lean manufacturing system with rework under fuzzy demand. Mathematics 7 (2018) 13.

[40] M. Tayyab, B. Sarkar and M. Ullah, Sustainable lot size in a multi-stage lean-green manufacturing process under uncertainty. Mathematics 7 (2018) 20.

[41] S. Tiwari, C.K. Jaggi, A.K. Bhunia, A.A. Shaikh and M. Goh, Two-warehouse inventory model for non-instantaneous deteriorating items with stock-dependent demand and inflation using particle swarm optimization. Ann. Oper. Res. 254 (2017) 401-423.

[42] Y.-C. Tsao and G.-J. Sheen, Effects of promotion cost sharing policy with the sales learning curve on supply chain coordination. Comput. Oper. Res. 39 (2012) 1872-1878.

[43] M. Ullah, I. Asghar, M. Zahid, M. Omair, A. AlArjani and B. Sarkar, Ramification of remanufacturing in a sustainable three-echelon closed-loop supply chain management for returnable products. J. Cleaner Prod. 290 (2021) 125609.

[44] H.-M. Wee, C.-C. Lo and P.-H. Hsu, A multi-objective joint replenishment inventory model of deteriorated items in a fuzzy environment. Eur. J. Oper. Res. 197 (2009) 620-631.

[45] J.C.P. Yu, Optimizing a two-warehouse system under shortage backordering, trade credit, and decreasing rental conditions. Int. J. Prod. Econ. 209 (2019) 147-155.

[46] Q. Zhang, W. Tang and J. Zhang, Green supply chain performance with cost learning and operational inefficiency effects. J. Cleaner Prod. 112 (2016) 3267-3284. 